TRANSACTIONS OF THE

AMERICAN MATHEMATICAL SOCIETY

Volume 183, September 1973

\title{
TORSION IN $K$-THEORY AND THE BOTT MAPS
}

\author{
BY
}

\section{ALBERT T. LUNDELL( $\left.{ }^{1}\right)$}

ABSTRACT. The nonstable Bott maps $b_{n}^{\prime}: U(n) \rightarrow \Omega^{2} U(n+1)$ for the unitary group are studied as to their behavior under iteration. They are then used to define and compute the coefficients of a spectrum. The corresponding cohomology theory is developed and compared with reduced complex $K$-theory. In this context the Chern character is induced by a map of spectra. The complex e-invariant appears as a coboundary in the long exact sequence of a cofibration.

In [11] the author defined maps $b_{n}^{\prime}: U(n) \rightarrow \Omega^{2} U(n+1)$ which are deformations of the classical Bott homotopy equivalence $b: U \rightarrow \Omega^{2} U$ [4], i.e., the composite $U(n) \rightarrow \Omega^{2} U(n+1) \rightarrow \Omega^{2} U$ is homotopic to the composite $U(n) \rightarrow U \rightarrow \Omega^{2} U$. The maps $b_{n}^{\prime}$ are natural with respect to the inclusions $U(k) \subset U(n)$ for $k \leq n$. The maps $b_{n}^{\prime}$ may be used to define homomorphisms $B_{n}: \pi_{r}(U(n)) \rightarrow \pi_{r+2}(U(n+1))$ as the composite homomorphism

$$
\pi_{r}(U(n)) \stackrel{b_{n^{*}}^{\prime}}{\longrightarrow} \pi_{r}\left(\Omega^{2} U(n+1)\right) \stackrel{\partial^{-2}}{\longrightarrow} \pi_{r+2}(U(n+1)) .
$$

The advantage gained by using the maps $B_{n}$ is that they give information on the nonstable homotopy of $U(n)$ not available from the classical Bott maps, and they agree with the classical results in the stable range. For example, the results of [11] show that the map $B_{n}: \pi_{r}(U(n)) \rightarrow \pi_{r+2}(U(n+1))$ is an isomorphism for $r \leq$ $2 n-1$, and $B_{n}: \pi_{2 n}(U(n)) \rightarrow \pi_{2(n+1)}(U(n+1))$ is a monomorphism.

This work applies these deformed Bott maps to the complex $K$-theory. The method is to define a spectrum using the maps $b_{n}^{\prime}$ and to develop the corresponding cohomology theory together with its relationships with $K$-theory and rational cohomology. The paper ends with an application which interprets the Adams $e$-invariant.

The paper is organized as follows. We first develop properties of the maps $B_{n}$ needed for later computations. We then construct a long exact sequence of cohomology theories analogous to the exact coefficient sequence in ordinary cohomology. $\$ \$ 3$ and 4 contain the definition of the spectrum, calculations of its homotopy and the interpretation of the resulting cohomology theory in terms of

Received by the editors April 11, 1972 and, in revised form, September 12, 1972.

AMS (MOS) subject classifications (1970). Primary 55B15, 55B20.

Key words and phrases. Bott map, spectrum, generalized cohomology, $K$-theory, Chern character, e-invariant. this work.

(1) The author was partially supported by NSF Grant GP29689 during preparation of

Copyright $\odot$ 1973, American Mathematical Society 
$K$-theory, rational cohomology, and the Chern character. The final sections contain some multiplicative properties of the cohomology theory, its behavior under the Adams operations, and an interpretation of the $e$-invariant.

Preliminary announcement of some of these results appeared in [12], although the reader should be cautioned that we have changed the indexing of the spectrum in this work for convenience in applications. Thus in [12], most of the results will differ from the ones here.

We work throughout in the category of finite $C W$ complexes with basepoint. We use $Q$ to denote the additive group of rational numbers, and $Z$ to denote the group of integers.

1. The Bott maps. For $n \geq 1$, let $U(n)$ be the unitary group, the group of complex $n \times n$ matrices with $A^{-1}=\bar{A}^{t}$ for $A \in U(n)$, and regard $U(n) \subset U(n+1)$ as the subgroup of matrices of the form $\left(\begin{array}{ll}1 & 0 \\ 0 & A\end{array}\right)$ for $A \in U(n)$. Let $U=U(\infty)=$ $\bigcup_{n \geq 1} U(n)$ and $U(0)=\{1\} \subset U(1)$. For $n \geq 1$, let $S U(n)$ be the subgroup of matrices of determinant 1. Clearly $S U(n) \subset S U(n+1)$ and we may define $S U=S U(\infty)=\bigcup_{n \geq 1} S U(n)$. We thus have chains of inclusions $U(0) \subset U(1) \subset \cdots \subset U(n) \subset \cdots \subset U(\infty)=U, U(0)$ $=S U(1) \subset S U(2) \subset \ldots \subset S U(n) \subset \cdots \subset S U(\infty)=S U \subset U$. It follows that for $0 \leq k \leq$ $l \leq m \leq \infty$, the Bott map [11] may be regarded as a map of triples

$$
b_{m}^{\prime}:(U(m), U(l), U(k)) \rightarrow\left(\Omega^{2} S U(m+1), \Omega^{2} S U(l+1), \Omega^{2} S U(k+1)\right) .
$$

Note that $b_{m}^{\prime}(U(0)) \subset \Omega^{2} S U(1)=\mathrm{pt}$.

Lemma 1.1. For $0<k \leq m \leq \infty$ there is a bomotopy ladder with commutative squares

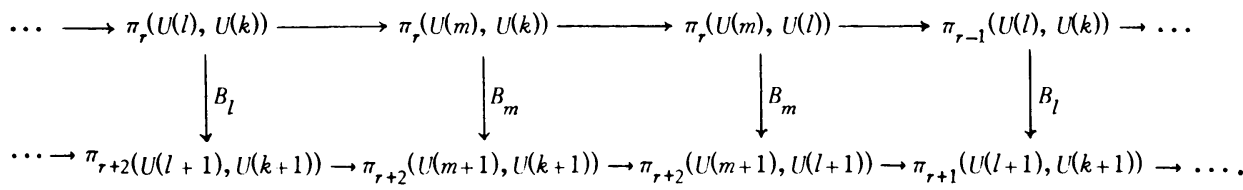

Proof. Let $B_{m}$ be the composite map

$B_{m}: \pi_{r}(U(m), U(k)) \stackrel{b_{m *}^{\prime}}{\longrightarrow} \pi_{r}\left(\Omega^{2} S U(m+1), \Omega^{2} S U(k+1)\right) \stackrel{\partial^{-2}}{\longrightarrow} \pi_{r+2}(S U(m+1), S U(k+1))$

$$
\cong \pi_{r+2}(U(m+1), U(k+1)),
$$

where $\partial^{2}: \pi_{r+2}(S U(m+1), S U(k+1)) \rightarrow \pi_{r}\left(\Omega^{2} S U(m+1), \Omega^{2} S U(k+1)\right)$ is the usual natural isomorphism.

In the case $k=0, m>0, B_{m}: \pi_{r}(U(m)) \rightarrow \pi_{r+2}(U(m+1))$.

Let $1 \leq r \leq n<\infty$ and let $\beta_{1, n}$ generate $\pi_{1}(U(n)) \cong Z$. Inductively define $\beta_{r, n}=B_{n-1}\left(\beta_{r-1, n-1}\right) \in \pi_{2 r-1}(U(n)) \cong Z$ for $2 \leq r \leq n$, i.e., $\beta_{r, n}=B_{n-1} B_{n-2}$ $\cdots B_{n-r+1}\left(\beta_{1, n-r+1}\right)$. According to [11, Proposition 3.2], $\beta_{r, n}$ generates $\pi_{2 r-1}(U(n))$. From naturality of the Bott maps $B$, if $i_{k}: U(n) \rightarrow U(n+k)$ is the 
inclusion, $i_{k} *\left(\beta_{r, n}\right)=\beta_{r, n+k}$, and if $i: U(n) \rightarrow U$ is the inclusion, $i_{*}\left(\beta_{r, n}\right)=\beta_{r}$, a generator of $\pi_{2 r-1}(U) \cong Z$.

Let $\iota_{2 n-1} \in \pi_{2 n-1}\left(s^{2 n-1}\right)$ be the class of the identity map, and let $\tau_{2 n-1} \epsilon$ $\pi_{2 n-1}(U(n), U(n-1))$ be such that $p_{*} \bar{\imath}_{2 n-1}=\iota_{2 n-1}$, where $p: U(n) \rightarrow S^{2 n-1}$ is the usual fibration. Finally, let $\gamma_{n-1}=\partial \tau_{2 n-1} \in \pi_{2(n-1)}(U(n-1)) \cong Z_{(n-1) !}$.

Proposition 1.2. For each $n \geq 1, B_{n}\left(\bar{\iota}_{2 n-1}\right)=n \epsilon_{n} \epsilon_{n+1} \bar{\iota}_{2 n+1}$ and $B_{n-1}\left(\gamma_{n-1}\right)$ $=n \epsilon_{n} \epsilon_{n+1} \gamma_{n}$, where $\epsilon_{k}= \pm 1$.

Proof. Consider the diagram

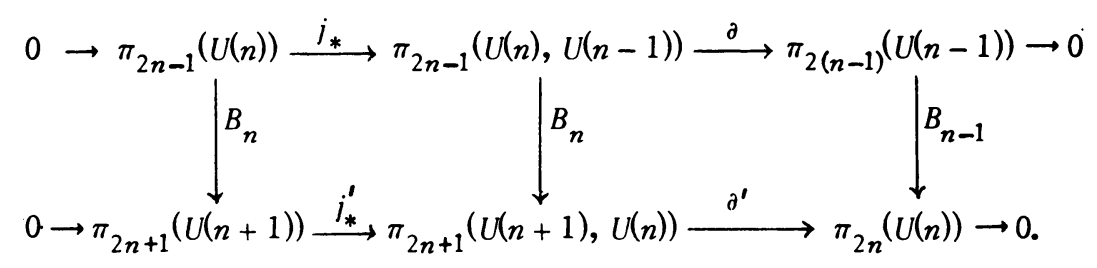

Since $\pi_{2(n-1)}(U(n-1)) \cong Z_{(n-1) !}, j_{*}\left(\beta_{n, n}\right)=\epsilon_{n}(n-1) ! \tau_{2 n-1}$, where $\epsilon_{n}= \pm 1$. Similarly $j_{*}^{\prime}\left(\beta_{n+1, n+1}\right)=\epsilon_{n+1} n ! \bar{\iota}_{2 n+1}$. Since $B_{n}\left(\beta_{n, n}\right)=\beta_{n+1, n+1}$, commutativity of the diagram yields $B_{n}\left(\tau_{2 n-1}\right)=n \epsilon_{n} \epsilon_{n+1} \bar{\iota}_{2 n+1}$ and $B_{n-1}\left(\gamma_{n-1}\right)=n \epsilon_{n} \epsilon_{n+1} \gamma_{n}$.

Corollary 1.3. For $n \geq 1$ and $r \geq 0, B_{n+r} B_{n+r-1} \cdots B_{n}\left(\iota_{2 n-1}\right)=$ $\epsilon_{n} \epsilon_{n+r}((n+r) ! /(n-1) !) \tau_{2(n+r)-1}$ and $B_{n+r-1} B_{n+r-2} \cdots B_{n-1}\left(\gamma_{n-1}\right)=$ $\epsilon_{n} \epsilon_{n+r}((n+r) ! /(n-1) !) \gamma_{n+r-1}$.

We next investigate the effect of the Bott maps on composition elements. We need two technical results which are easily proved using standard homotopy theory arguments.

Proposition 1.4. Let $(X, A, B)$ be a triple, let $\alpha \in \pi_{n}(X, A)$, and let $\beta=$ $S \beta^{\prime} \in \pi_{r+1}\left(D^{n}, S^{n-1}\right)$. Then $\partial(\alpha \circ \beta)=\partial \alpha \circ \beta^{\prime}$.

Corollary 1.5. If $(X, A)$ is a pair $\alpha \in \pi_{n}(X, A)$ and $S \beta \in \pi_{r+1}\left(D^{n}, S^{n-1}\right)$, $\partial(\alpha \circ S \beta)=\partial \alpha \circ \beta \in \pi_{r}(\Omega X, \Omega A)$.

We now apply these technical results to the Bott maps.

Proposition 1.6. If $\theta \circ \zeta \in \pi_{r}(U(n), U(k))$ where $\zeta \in \pi_{r}\left(D^{m}, S^{m-1}\right)$ and $\theta \in \pi_{m}(U(n), U(k))$, then $B_{n}(\theta \circ \zeta)=B_{n}(\theta) \circ S^{2} \zeta$.

Proof. The map $\partial^{2}: \pi_{r+2}(U(n+1), U(k+1)) \rightarrow \pi_{r}\left(\Omega^{2} U(n+1), \Omega^{2} U(k+1)\right)$ is an isomorphism and $\partial^{2}\left(B_{n}(\theta) \circ S^{2} \zeta\right)=\partial^{2} B_{n}(\theta) \circ \zeta=b_{n *}^{\prime} \theta \circ \zeta=b_{n *}^{\prime}(\theta \circ \zeta)=$ $\partial^{2} B_{n}(\theta \circ \zeta)$ 
The following theorem gives results on the nontriviality or vanishing of iterates of the Bott maps.

Theorem 1.7. (i) The Bott map $B_{n}: \pi_{s}(U(n), U(m)) \rightarrow \pi_{s+2}(U(n+1), U(m+1))$ is nontrivial for $s=2 m-1+2 k$ if $0 \leq k \leq n-m$ and $0<m<n \leq \infty$.

(ii) The Bott map $B_{n}: \pi_{s}(U(n)) \rightarrow \pi_{s+2}(U(n+1))$ is nontrivial for $s=$ $2(n+k)$ if $0 \leq k<n$ and $1<n<\infty$.

(iii) The Bott map $B_{n}: \pi_{s}(U(n)) \rightarrow \pi_{s+2}(U(n+1))$ is an isomorpbism for $s=$ $2 k-1$ if $0<k \leq n$ and $0<n \leq \infty$.

(iv) If $0<k, 0<m<n<\infty$ and $s=2 n-1+k$, or $0 \leq k, 0<m<n \leq \infty$ and $s=2 m+2 k$, then for some $r>0$, an r-fold iterate of the Bott map vanishes on $\pi_{s}(U(n), U(m))$.

(v) If $0<k, 1<n<\infty$, and $s=2 n-1+2 k$, then for some $r>0$ an r-fold iterate of the Bott map vanishes on $\pi_{s}(U(n))$.

The only cases left undecided are whether Bott maps are nontrivial or iterates are trivial on the groups $\pi_{2(n+k)}(U(n))$ for $k \geq n$, since in all other cases not mentioned in the theorem, the groups themselves are trivial. The author does not know what the effect of the Bott map is in these undecided cases, although using calculations of Sigrist [13] one can prove $0=B_{2}: \pi_{8}(U(2)) \rightarrow \pi_{10}(U(3))$ and $0 \neq$ $B_{3}: \pi_{12}(U(3)) \rightarrow \pi_{14}(U(4))$.

Part (iii) of the theorem is included only for completeness and will not be proved here, since it is assumed in earlier remarks and proved in [11]. Proofs of the other parts are in the following sequence of propositions.

Proposition 1.8. For $0<k \leq n-m$ and $0<m<n \leq \infty$, the Bott maps $B_{n}$ : $\pi_{2 m-1+2 k}(U(n), U(m)) \rightarrow \pi_{2 m+1+2 k}(U(n+1), U(m+1))$ are nontrivial.

Proof. One easily checks that

$$
j_{*}^{\prime}: \pi_{2 m-1+2 k}(U(n), U(m)) \rightarrow \pi_{2 m-1+2 k}(U(n), U(m+k-1))
$$

is nontrivial, since both groups are of rank 1 and the map $j_{*}^{\prime}$ has finite kernel and cokernel; so if we prove $B_{n}: \pi_{2 m-1+2 k}(U(n), U(m+k-1)) \rightarrow$ $\pi_{2 m+1+2 k}(U(n+1), U(m+k))$ is nontrivial, the result follows by naturality of the maps $B_{n}$. Since $2 m-1+2 k-1<2 n-2, \partial^{\prime}: \pi_{2 m-1+2 k}(U(n), U(m+k-1)) \rightarrow$ $\pi_{2 m-1+2 k-1}(U(m+k-1))$ is onto, and $B_{m+k-1}: \pi_{2 m-2+2 k}(U(m+k-1)) \rightarrow$ $\pi_{2 m+2 k}(U(m+k))$ is a monomorphism $B_{m+k-1}\left(\gamma_{m+k-1}\right)= \pm(m+k) \gamma_{m+k}$. Since $\partial B_{n}=B_{m+k-1} \partial^{\prime}$ and $B_{m+k-1} \partial^{\prime}$ is nontrivial, $B_{n}$ is nontrivial and the result follows.

Proposition 1.9. For $0 \leq k<n$ and $1<n<\infty$, the Bott map $B_{n}: \pi_{2(n+k)}(U(n))$ $\rightarrow \pi_{2(n+k)+2}(U(n+1))$ is nontrivial. 
Proof. Since $\pi_{2(n+k)}(U(n+k))=Z_{(n+k) !}$ is cyclic, the cokernel of the map $i_{*}: \pi_{2(n+k)}(U(n)) \rightarrow \pi_{2(n+k)}(U(n+k))$ is cyclic of order $\delta_{n, k}$. By using the fibrations over spheres associated with the inclusions $U(n) \subset U(n+1) \subset \cdots \subset U(n+k)$ and knowledge of stable homotopy of spheres, one estimates [12, Proposition 2.3] that for $p$ an odd prime $v_{p}\left(\delta_{n, k}\right) \leq[k /(p-1)]$ if $k \leq p(p-1)$, where $v_{p}(n)$ is the highest power of $p$ dividing $n$. We will show $i_{*}$ is nontrivial by showing $v_{p}\left(\delta_{n, k}\right)<v_{p}((n+k) !)$ for $k<n$ and a suitable prime $p$. Since $B_{n+k}: \pi_{2(n+k)}(U(n+k))$ $\rightarrow \pi_{2(n+k+1)}(U(n+k+1))$ is a monomorphism by Proposition 1.2, and the Bott maps are natural with respect to inclusions $i_{*}^{\prime} B_{n}=B_{n+k}{ }^{i}$ is nontrivial so $B_{n}$ is nontrivial.

To see $v_{p}\left(\delta_{n, k}\right)<v_{p}((n+k) !)$, recall that by Bertrand's postulate [14], there is a prime $p$ satisfying $k+1<p \leq 2(k+1)$, and if $k \geq 1 p$ is odd, so $k+1<p$ $\leq 2 k+1 \leq n+k$. Moreover $k<p<p(p-1)$, so $v_{p}\left(\delta_{n, k}\right)=[k /(p-1)]$. But then $k<p-1$, so $v_{p}\left(\delta_{n, k}\right)=0$. On the other hand, $v_{p}((n+k) !) \geq[(n+k) / p] \geq$ $[(2 k+1) / p] \geq 1$. Thus $v_{p}\left(\delta_{n, k}\right)<v_{p}((n+k) !)$.

If $\alpha \in \pi_{m+r}\left(S^{m}\right)$, let $o(\alpha)$ be the order of $\alpha$. Let $o_{m, r}=\sup \left\{d(\alpha) \mid \alpha \epsilon \pi_{m+r}\left(S^{m}\right)\right.$ and $o(\alpha)<\infty\}$. By the Freudenthal suspension the orem $o_{m, r}=o_{m+1, r}=\cdots$ for $r \leq m-2$. James [8] and Bousfield [5] have results on upper bounds for the numbers $o_{m, r}$. Recall that if $\alpha \in \pi_{m+r}\left(S^{m}\right)$ and $o(\alpha)<\infty$, then $o(\alpha)$ divides $o_{m, \dot{r}}$

Proposition 1.10. Let $k \geq 1$ and $a \in \pi_{2 n-1+k}(U(n), U(n-1))$ be such that $p_{*}(\alpha)=S \alpha^{\prime} \in \pi_{2 n-1+k}\left(S^{2 n-1}\right)$. If o $\left(S^{2 r+1} \alpha^{\prime}\right)$ divides $(n+r) ! /(n-1) !$, then $B_{n+r-1} \cdots B_{n}(\alpha)=0$.

Proof. Since $p_{*}(\alpha)=S \alpha^{\prime}=\iota_{2 n-1} \circ S \alpha^{\prime}$, we have $\alpha=\tau_{2 n-1} \circ C \alpha^{\prime}$, where $C \alpha^{\prime} \in \pi_{2 n-1+k}\left(D^{2 n-1}, S^{2 n-1}\right)$ is the cone on $\alpha^{\prime}$. Thus

$$
\begin{aligned}
B_{n+r-1} \cdots B_{n}(\alpha) & =B_{n+r-1} \cdots B_{n}\left(\iota_{2 n-1}\right) \circ S^{2 r} C \alpha^{\prime} \\
& = \pm((n+r) ! /(n-1) !) \bar{\iota}_{2(n+r)-1} \circ S^{2 r} C \alpha^{\prime}
\end{aligned}
$$

It follows that $p_{*} B_{n+r-1} \cdots B_{n}(\alpha)= \pm((n+r) ! /(n-1) !) S^{2 r+1} p_{*}(\alpha)=0$, and since $p_{*}$ is an isomorphism, $B_{n+r-1} \cdots B_{n}(\alpha)=0$.

Corollary 1.11. If $k \geq 1$, then for some $r \geq 1$,

$$
B_{n+r-1} \cdots B_{n} \pi_{2 n-1+k}(U(n), U(n-1))=0 \text {. }
$$

Proof. For $1 \leq k \leq 2 n-3, p_{*} \pi_{2 n-1+k}(U(n), U(n-1))=\pi_{2 n-1+k}\left(S^{2 n-1}\right)=$ $S \pi_{2 n-2+k}\left(S^{2 n-2}\right)$. If we choose $r$ so large that $o_{2 n-1, k} \operatorname{divides}(n+r) ! /(n-1)$ !, then $B_{n+r-1} \cdots B_{n} \pi_{2 n-1+k}(U(n), U(n-1))=0$. If $2 n-2 \leq k$, observe that $p_{*} B_{n+s-1} \cdots B_{n} \pi_{2 n-1+k}(U(n), U(n-1)) \subset \pi_{2(n+s)-1+k}\left(s^{2(n+s)-1}\right)=$ $S \pi_{2(n+s)-2+k}\left(s^{2(n+s)-2}\right)$ for $k \leq 2(n+s)-3$ or $s \geq(k+3) / 2-n$. Now choose 
$r$ so that $o_{2(n+s)-1, k}$ divides $(n+s+r) ! /(n+s-1)$ ! Then $B_{n+s+r-1} \cdots$ $B_{n} \pi_{2 n-1+k}(U(n), U(n-1))=0$.

Corollary 1.12. If $k \geq 1$ and $0<m<n<\infty$, then for some $r>0, B_{n+r-1} \cdots$ $B_{n} \pi_{2 n-1+k}(U(n), U(m))=0$.

Proof. By induction on $n-m$. The previous corollary is the case $n-m=1$. Suppose the proposition is true for $1 \leq n-m<l$ and consider the diagram

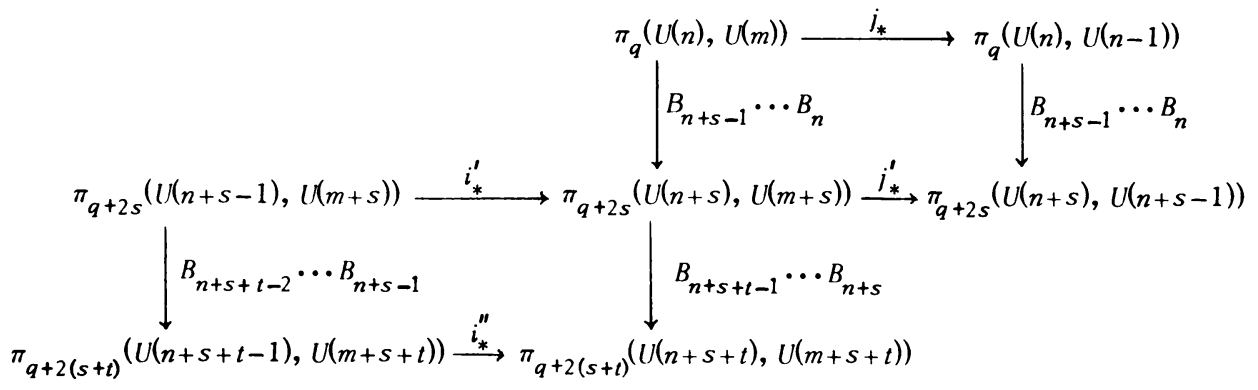

where $q=2 n-1+k$ and $n-m=l \geq 2$. By the previous corollary, if $a \epsilon$ $\pi_{q}(U(n), U(m))$, we may choose $s>1$ so that $0=B_{n+s-1} \cdots B_{n} j_{*} \alpha=j_{*}^{\prime} B_{n+s-1} \cdots B_{n}(\alpha)$. By exactness of the middle row, $B_{n+s-1} \cdots B_{n}(\alpha)=i_{*}^{\prime}(\beta)$. But $n+s-1-(m+s)$ $=n-m-1<l$, and by the inductive hypothesis we may choose $t$ so that $B_{n+s+t-2} \cdots B_{n+s-1}(\beta)=0$. Thus $B_{n+s+t-1} \cdots B_{n}(\alpha)=B_{n+s+t-1} \cdots B_{n+s} i_{*}^{\prime} \beta$ $=i_{*}^{\prime \prime} B_{n+s+t-2} \cdots B_{n+s+t-1}(\beta)=0$. Let $r=s+t$.

Entirely analogous inductive proofs give the following two corollaries.

Corollary 1.13. If $0 \leq k$ and $0<m<n \leq \infty$, then for some $r>0, B_{n+r-1} \cdots$ $B_{n} \pi_{2 m+2 k}(U(n), U(m))=0$.

Corollary 1.14. If $0 \leq k$ and $1<n<\infty$, then for some $r>0, B_{n+r-1} \cdots$ $B_{n} \pi_{2 n-1+2 k}(U(n))=0$.

These propositions complete the proof of the theorem.

The following two propositions are of a technical nature and are for later use. We will see later that they are related to the Bott periodicity maps.

Proposition 1.15. Let $0<k \leq n-m$ and $0<m<n<\infty$. Then for $0<s<m$ and some $r>0$ there is a bomomorphism $b$ such that the following diagram commutes:

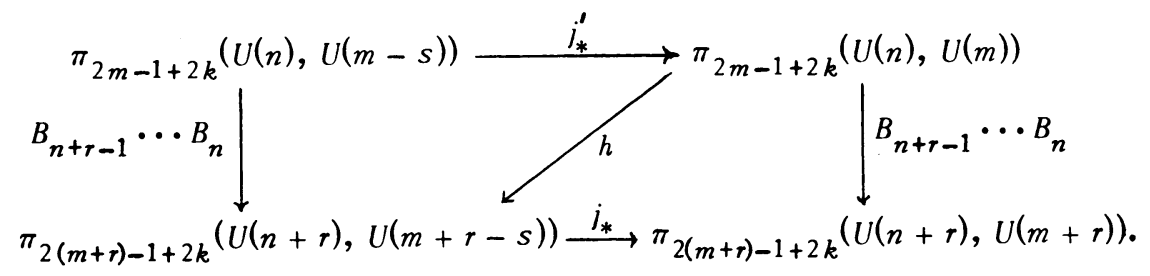


Proof. Consider the commutative diagram

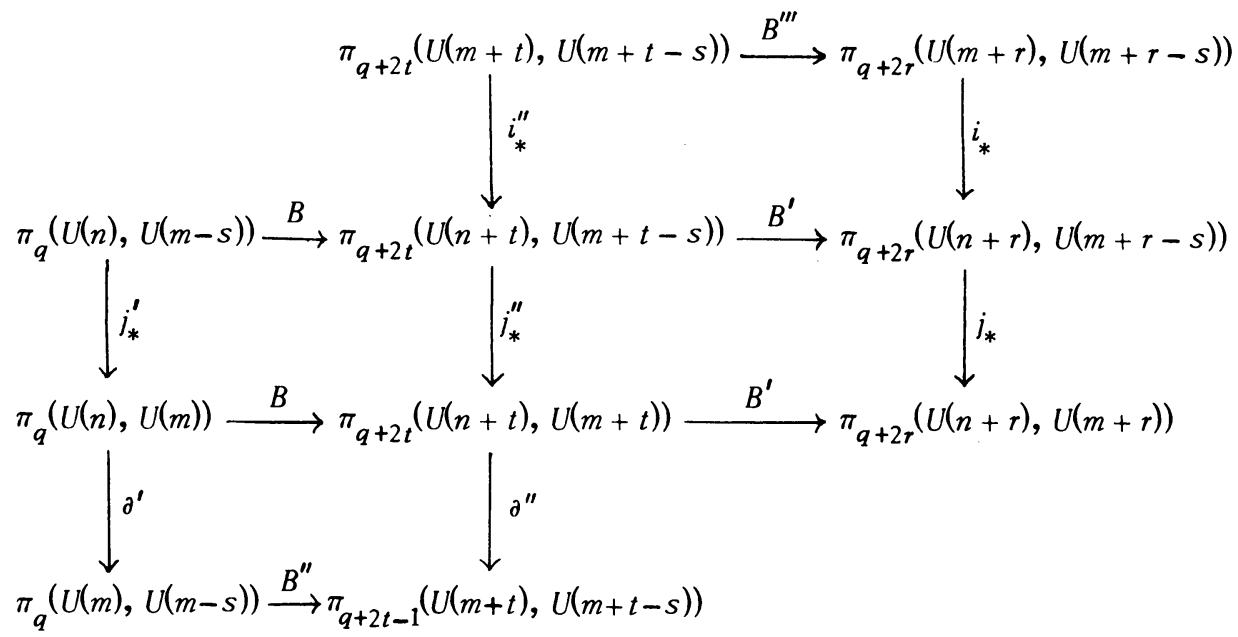

where $q=2 m-1+2 k, B=B_{n+t-1} \cdots B_{n}, B^{\prime}=B_{n+r-1} \cdots B_{n+t}, B^{\prime \prime}=$ $B_{m+t-1} \cdots B_{m}$, and $B^{\prime \prime \prime}=B_{m+r-1} \cdots B_{m+t}$ with $r$ to be chosen. Now $q-1$ is even, and by Corollary 1.13, we may choose $t>0$ so that $B^{\prime \prime}=0$. Thus $\partial^{\prime \prime} B=0$ and $\operatorname{Im} B \subset \operatorname{Im} j_{*}^{\prime \prime}$. For each $a \in \pi_{q}(U(n), U(m))$ choose

$$
b^{\prime}(\alpha) \in \pi_{q+2 t}(U(n+t), U(m+t-s))
$$

such that $j_{*}^{\prime \prime} b^{\prime}(\alpha)=B(\alpha)$. If $b^{\prime \prime}(\alpha)$ is another such choice $b^{\prime}(a)$ $b^{\prime \prime}(\alpha) \in \operatorname{Im} i_{*}^{\prime \prime}$. Choose $r>0$ so that $B^{\prime \prime \prime}=0$, which is possible by Corollary 1.13, since $q+2 t=2 m-1+2 k+2 t>2(m+t)-1$. Thus $B^{\prime} b^{\prime}=B^{\prime} b^{\prime \prime}$, and if we set $b=B^{\prime} b^{\prime}$, one easily sees that $b$ is a homomorphism and $j_{*} b=B^{\prime} B$. For $\beta \in \pi_{q}(U(n), U(m-s))$, we have $B(\beta)-b^{\prime} j_{*}^{\prime}(\beta) \in \operatorname{Im} i_{*}^{\prime \prime}$, so $B^{\prime} B(\beta)=b j_{*}^{\prime}(\beta)$.

Proposition 1.16. Let $0 \leq k$ and $0<n<\infty$. Then for $0<s<n$ and some $r>0$, there is a bomomorphis $m$ such that the following diagram commutes:

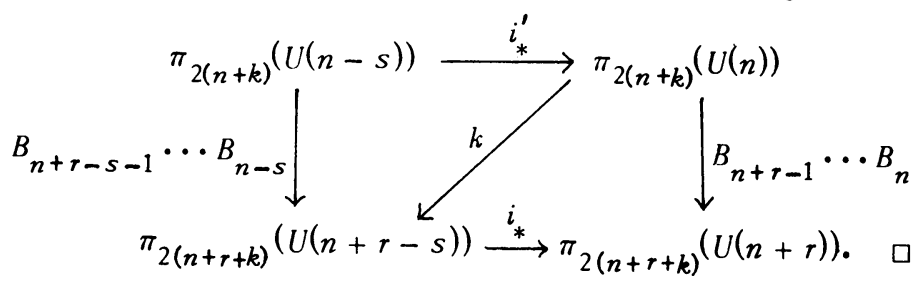

The following is an application of Proposition 1.2.

James [9] defines numbers $W\{n, k\}$ as the order of the cokernel of $j_{*}$ in the exact sequence

$$
\pi_{2 n-1}(U(n), U(n-k)) \stackrel{j_{*}}{\longrightarrow} \pi_{2 n-1}(U(n), U(n-1)) \rightarrow \pi_{2 n-2}(U(n-1), U(n-k)),
$$

i.e., $j_{*} \pi_{2 n-1}(U(n), U(n-k))=W\{n, k\} \pi_{2 n-1}(U(n), U(n-1))$. Also in [9] James derives several divisibility relations among the $W\{n, k\}$. The numbers $W\{n, k\}$ are 
known for $k \leq 4$ by the work of Eckmann [7] and Sigrist [131. The following is evidently a new divisibility relation on the James numbers.

Theorem 1.17. For $n>k, W\{n+1, k\}$ divides $n W\{n, k\}$.

Proof. Consider the diagram

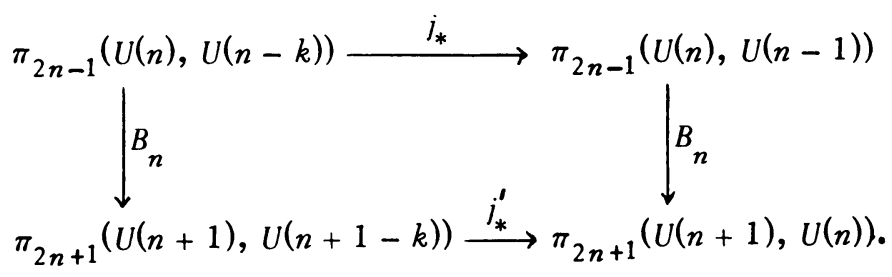

We have $n W\{n, k\} \pi_{2 n+1}(U(n+1), U(n))=\operatorname{Im} B_{n} j_{*}=\operatorname{Im} j_{*}^{\prime} B_{n} \subset \operatorname{Im} j_{*}^{\prime}=$ $W\{n+1, k\} \pi_{2 n+1}(U(n+1), U(n))$. Thus $W\{n+1, k\}$ divides $n W\{n, k\}$, since $\pi_{2 n+1}(U(n+1), U(n)) \cong Z$.

Corollary 1.18. $W\{n+r, k\}$ divides $((n+r-1) ! /(n-1) !) W\{n, k\}$, for $n>k$ and $r>0$.

2. Relative spectra. Let $(E, F)$ be a pair of spectra, i.e., for each integer $k$, there is an inclusion $i_{k}: F_{k} \rightarrow E_{k}$ such that the following diagram homotopy commutes:

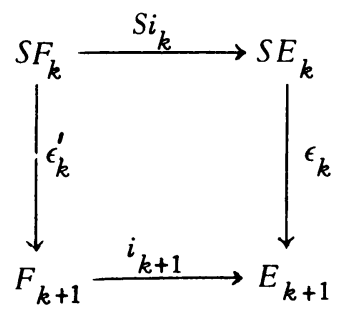

The homotopy groups of $(\mathrm{E}, \mathrm{F})$ are defined to be the direct limit

$$
\pi_{n}(\mathbf{E}, \mathbf{F})=\lim _{k \rightarrow \infty}\left\{\pi_{n+k}\left(E_{k}, F_{k}\right), \phi_{k}\right\}
$$

where $\phi_{k}$ is the composite map

$$
\pi_{n+k}\left(E_{k}, F_{k}\right) \stackrel{S}{\longrightarrow} \pi_{n+k+1}\left(S E_{k}, S F_{k}\right) \stackrel{\epsilon_{k *}}{\longrightarrow} \pi_{n+k+1}\left(E_{k+1}, F_{k+1}\right) .
$$

Since direct limits preserve exactness, we obtain the following.

Proposition 2.1. If (E, F) is a pair of spectra there is a long exact sequence

$$
\cdots \rightarrow \pi_{n}(\mathrm{~F}) \stackrel{\mathrm{i}_{*}}{\longrightarrow} \pi_{n}(\mathrm{E}) \stackrel{\mathrm{j}_{*}}{\longrightarrow} \pi_{n}(\mathrm{E}, \mathrm{F}) \stackrel{\partial_{*}}{\longrightarrow} \pi_{n-1}(\mathrm{~F}) \rightarrow \cdots
$$

Define cohomology groups $b^{n}(X ; \mathbf{E}, \mathbf{F})=\lim _{k \rightarrow \infty}\left[C S^{k-n-1} X, S^{k-n-1} X ; E_{k}, F_{k}\right]$ with respect to the maps 


$$
\begin{aligned}
& {\left[C S^{k-n-1} X, S^{k-n-1} X ; E_{k}, F_{k}\right] } \stackrel{S}{\longrightarrow}\left[C S^{k-n} X, S^{k-n} X ; S E_{k}, S F_{k}\right] \\
& \stackrel{\epsilon_{k *}}{\longrightarrow}\left[C S^{k-n} X, S^{k-n} X ; E_{k+1}, F_{k+1}\right],
\end{aligned}
$$

where the homotopy sets are homotopy classes of based maps.

We thus define a cohomology theory and remark that we will obtain a reduced theory from these definitions.

Using relative spectra we obtain exact sequences analogous to coefficient sequences in ordinary cohomology theory.

Theorem 2.2. If $(\mathbf{E}, \mathbf{F})$ is a pair of spectra there is a long exact sequence

$$
\cdots \rightarrow b^{n}(X ; \mathbf{F}) \rightarrow b^{n}(X ; \mathbf{E}) \rightarrow b^{n}(X ; \mathbf{E}, \mathbf{F}) \rightarrow b^{n+1}(X ; \mathbf{F}) \rightarrow \cdots
$$

3. The nonstable unitary spectrum. For our purposes it will be more convenient to use the adjoint notion of a spectrum, i.e., if $\mathbf{E}$ is a spectrum we will take our maps to be adjoints of the usual ones, so $\epsilon_{k}: E_{k} \rightarrow \Omega E_{k+1}$.

The nonstable unitary spectrum $\mathbf{T U}$ is defined as follows:

$$
\begin{array}{cl}
T U_{2 k-1}=U(k), & k>0, \\
T U_{2 k}=\Omega U(k+1), & k \geq 0, \\
T U_{m}=\text { point, } & m<0, \\
\tau_{2 k-1}=b_{k}^{\prime}: T U_{2 k-1}=U(k) \longrightarrow & \Omega^{2} U(k+1)=\Omega T U_{2 k}, \\
\tau_{2 k}=\mathrm{id}: T U_{2 k}=\Omega U(k+1) \rightarrow \Omega U(k+1)=\Omega T U_{2 k+1},
\end{array}
$$

where $b_{k}^{\prime}: U(k) \rightarrow \Omega^{2} U(k+1)$ is the Bott map of [11]. Note that $T U_{2 k-1}=U(k)$ is the homotopy type of $\Omega B U(k)$, where $B U(k)$ is the universal classifying space for $U(k)$.

Remark. This is a change in indexing from the announcement [12]. The result will be a change of indexing in Theorems 1,4 , and $\$ 3$ of [12], but is much more convenient for later applications.

Recall that the spectrum $\mathrm{BU}$ is defined by

$$
\begin{gathered}
B U_{2 k-1}=U, \quad B U_{2 k}=\Omega U, \\
\beta_{2 k-1}=b: B U_{2 k-1}=U \rightarrow \Omega^{2} U=\Omega B U_{2 k}, \\
\beta_{2 k}=\mathrm{id}: B U_{2 k}=\Omega U \rightarrow \Omega U=\Omega B U_{2 k+1},
\end{gathered}
$$

where $b: U \rightarrow \Omega^{2} U$ is the original homotopy equivalence of Bott [4]. Again, $B U_{2 k}=\Omega U$ is homotopy equivalent to the universal classifying space $B U$. 
According to [11], the diagram

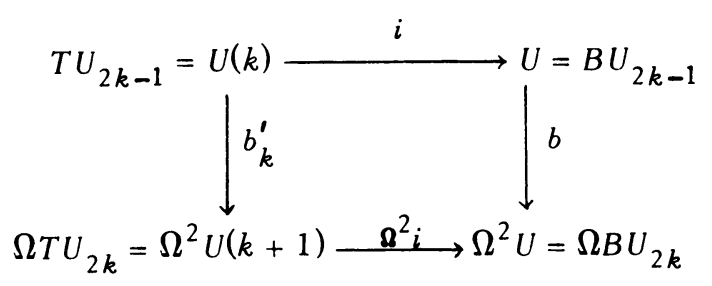

homotopy commutes, where $i: U(k) \rightarrow U$ is the inclusion, so ( $\mathbf{B U}, \mathbf{T U})$ is a relative spectrum.

Theorem 3.1. (i) The bomotopy groups of TU are as follows:

$$
\pi_{n}(\mathrm{TU})=\left\{\begin{array}{cl}
Z & \text { if } n \text { is even, } n \leq 0, \\
Q / Z & \text { if } n \text { is odd, } n>0 \\
0 & \text { otherwise. }
\end{array}\right.
$$

(ii) The homotopy groups of (BU, TU) are as follows:

$$
\pi_{n}(\mathbf{B U}, \mathbf{T U})= \begin{cases}Q & \text { if } n \text { is even, } n>0, \\ 0 & \text { otherwise. }\end{cases}
$$

(iii) The bomotopy groups of $\mathrm{BU}$ are as follows:

$$
\pi_{n}(\mathbf{B U})=\left\{\begin{array}{l}
Z \text { if } n \text { is even, } \\
0 \text { if } n \text { is odd. }
\end{array}\right.
$$

Proof. Part (iii) is well known and is included here only for completeness.

We first remark that since $\pi_{n}(E, F)=\lim _{k \rightarrow \infty}\left\{\pi_{n+k}\left(E_{k}, F_{k}\right), \phi_{k}\right\}$, and since the odd integers are cofinal in the integers

$$
\pi_{n}(\mathrm{E}, \mathrm{F})=\lim _{k \rightarrow \infty}\left\{\pi_{n+2 k-1}\left(E_{2 k-1}, F_{2 k-1}\right), \phi_{2 k} \phi_{2 k-1}\right\} .
$$

Thus in the cases in which we are interested here

$$
\pi_{n}(\mathrm{TU})=\lim _{k \rightarrow \infty}\left\{\pi_{n+2 k-1}(U(k)), B_{k}\right\}, \quad \pi_{n}(\mathbf{B U}, \mathrm{TU})=\lim _{k \rightarrow \infty}\left\{\pi_{n+2 k-1}(U, U(k)), B\right\} .
$$

Part (i). For $n \leq 0$, the maps $B_{k}$ are all isomorphisms by part (iii) of Theorem 1.7. Since we are in the stable range for $U(k), \pi_{n}(\mathrm{TU})=0$ or $Z$ if $n$ is odd or even respectively.

For $n \geq 0$ and even, part (v) of Theorem 1.7 insures that certain iterates of the maps $B_{k}$ are trivial, hence $\pi_{n}(\mathrm{TU})=0$.

For $n=2 s+1 \geq 1$, consider the exact sequences

$$
\begin{aligned}
\pi_{2(k+s)+1}(U(k+s), U(k)) & \rightarrow \pi_{2 k-1+2 s+1}(U(k)) \stackrel{i_{*}}{\longrightarrow} \pi_{2(k+s)-1+1}(U(k+s)) \\
& \rightarrow \pi_{2(k+s)}(U(k+s), U(k)) .
\end{aligned}
$$


By Theorem 1.7, part (iv), the limits of the groups on the ends with respect to the Bott maps are trivial, so the maps $i_{*}$ induce an isomorphism

$$
i_{*}: \pi_{2 s+1}(\mathrm{TU}) \rightarrow \pi_{1}(\mathrm{TU}) \text {. }
$$

In fact, the maps $k$ of Proposition 1.16 provide an inverse for $i_{*}$ in the limit.

It remains to calculate $\pi_{1}$ (TU). To do this, define monomorphisms $\phi_{k}$ : $\pi_{2 k}(U(k)) \rightarrow Q / Z$ by $\phi_{k}\left(\gamma_{k}\right)=\left[\epsilon_{k+1} / k !\right] \in Q / Z$. For each $k \geq 2$ the diagram

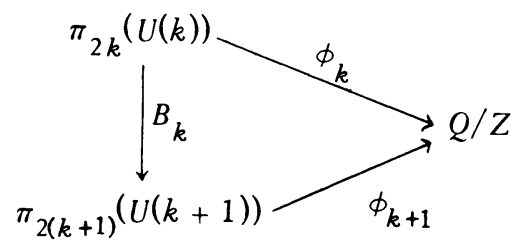

commutes, and we have an induced monomorphism $\Phi: \pi_{1}$ (TU) $\rightarrow Q / Z$. If $[p / q] \epsilon$ $Q / Z$, then $[p / q]=[p(q-1) ! / q !]=\phi_{q}\left(\epsilon_{q+1} p(q-1) ! \gamma_{q}\right)$, so $\Phi$ is an epimorphism.

The proof of part (ii) is similar. For $n \leq 1, \pi_{n+2 k-1}(U, U(k))=0$, so in the limit with respect to the Bott maps, $\pi_{n}(\mathbf{B U}, \overline{\mathrm{TU}})=0$.

If $n=2 s-1>1$, iterates of the Bott maps eventually vanish on $\pi_{2 k-1+2 s-1}(U, U(k))$ by Theorem 1.7, part (iv). Thus $\pi_{2 s-1}(\mathbf{B U}, \mathbf{T U})=0$.

If $n=2 s>0$, Theorem 1.7, part (iv), together with the exact sequences

$$
\begin{aligned}
& \pi_{2 k+2 s-1}(U(k+s-1), U(k)) \rightarrow \pi_{2 k-1+2 s}(U, U(k)) \\
& \stackrel{j_{*}}{\longrightarrow} \pi_{2(k+s-1)-1+2}(U, U(k+s-1)) \rightarrow \pi_{2(k+s-1)}(U(k+s-1), U(k))
\end{aligned}
$$

yield an isomorphism $j_{*}: \pi_{2 s}(\mathbf{B U}, \mathbf{T U}) \rightarrow \pi_{2}(\mathbf{B U}, \mathbf{T U})$. As before the maps $b$ of Proposition 1.15 provide an inverse for $j_{*}$ in the limit.

To calculate $\pi_{2}(\mathrm{BU}, \mathrm{TU})$, note that the diagram

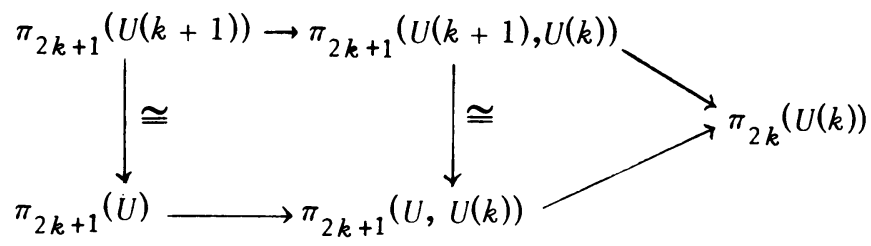

is commutative. Thus it is sufficient to calculate $\lim _{k \rightarrow \infty} \pi_{2 k+1}(U(k+1), U(k))$ with respect to the Bott maps. As in the calculation for part (i) of this theorem, define monomorphisms $\psi_{k}: \pi_{2 k+1}(U(k+1), U(k)) \rightarrow Q$ by $\psi_{k}\left(\bar{\iota}_{2 k+1}\right)=\epsilon_{k+1} / k !$. These maps are compatible with the Bott maps and induce an isomorphism

$$
\Psi: \lim _{k \rightarrow \infty}\left\{\pi_{2 k+1}(U(k+1), U(k)), B_{k+1}\right\} \rightarrow Q .
$$

This establishes $\pi_{2}(\mathbf{B U}, \mathbf{T U}) \cong Q$. 
Remarks. From the definitions of the maps $\Phi, \Psi$ in this proof, one easily sees that, for $s>0$, the exact sequence

$$
0 \rightarrow \pi_{2 s}(\mathrm{BU}) \rightarrow \pi_{2 s}(\mathrm{BU}, \mathrm{TU}) \rightarrow \pi_{2 s-1}(\mathrm{TU}) \rightarrow 0
$$

is isomorphic to the exact sequence $0 \rightarrow Z \rightarrow Q \rightarrow Q / Z \rightarrow 0$ with the usual embedding $Z \subset Q$.

We also point out that the proof of this theorem only requires knowledge of the groups $\pi_{n}(U(k))$ for $n \leq 2 k, \pi_{n}\left(S^{2 k-1}\right)$ for $n \leq 2 k-1$, the $p$-primary part of the stable $i$ stem for $i<2 p(p-1)-2$, and the fact that for $r>0$ the groups $\pi_{2 k-1+r}\left(s^{2 k-1}\right)$ are finite.

4. The exact cohomology sequence. Since ( $B U, T U)$ is a relative spectrum, by Theorem 2.2 there is a long exact sequence

$$
\ldots b^{n}(X ; \mathbf{T U}) \stackrel{i_{*}}{\longrightarrow} b^{n}(X ; \mathbf{B U}) \stackrel{j_{*}}{\longrightarrow} b^{n}(X ; \mathbf{B U}, \mathbf{T U}) \stackrel{\beta}{\longrightarrow} b^{n+1}(X ; \mathrm{TU}) \rightarrow \ldots
$$

which we are now able to interpret.

First of all, $b^{n}(X ; \mathbf{B U})=\lim _{k \rightarrow \infty}\left\{\left[S^{2 k+1-n} X ; U\right], b_{*}\right\}$, where $b: U \rightarrow \Omega^{2} U$ is the classical Bott homotopy equivalence. Since we are using homotopy classes of based maps, we see that $b^{n}(X ; \mathbf{B U})=\widetilde{K}^{n}(X)$ and the cohomology theory $b^{*}(; \mathbf{B U})$ is reduced complex $K$-theory $\tilde{K}^{*}$.

Proposition 4.1. For each integer $n$,

$$
b^{n}(X ; \mathbf{B U}, \mathbf{T U}) \cong \sum_{r>0} \widetilde{H}^{n+2 r}(X ; Q),
$$

where $\tilde{H}^{*}(Q)$ is ordinary reduced singular cobomology with rational coefficients.

Proof. As in Atiyah and Hirzebruch [3] or Dyer [6], there is a spectral sequence for $b^{n}(X ; \mathbf{B U}, \mathbf{T U})$ with $E_{2}^{p_{0} q}=H^{p}\left(X ; \tilde{b}^{q}\left(S^{0} ; \mathbf{B U}, \mathbf{T U}\right)\right)=H^{p}\left(X ; \pi_{-q}(\mathbf{B U}, \mathbf{T U})\right)$, where $H^{*}$ is ordinary singular cohomology. Since $\pi_{-q}(\mathbf{B U}, \mathbf{T U})$ is a $Q$-module for all $q$, it follows that $b^{n}(X ; \mathbf{B U}, \mathbf{T U})$ is a $Q$-module for all $n$. According to Dyer [6, Theorem 4], there is a natural equivalence of cohomology theories

$$
\sum_{i+j=n} \pi_{S}^{i}(X) \otimes \tilde{b}^{j}\left(S^{0} ; \mathbf{B U}, \mathbf{T U}\right) \otimes Q \rightarrow b^{n}(X ; \mathbf{B U}, \mathbf{T U}) \otimes Q
$$

(the factor $Q$ is superfluous here since $b^{*}(; \mathbf{B U}, \mathbf{T U})$ is a $Q$-module) where $\pi_{S}^{i}(X)$ is a stable cohomotopy group. But $\pi_{S}^{i}(X) \otimes Q \cong \tilde{H}^{i}(X) \otimes Q$, so the equiv. alence reduces to

$$
\sum_{r>0} \widetilde{H}^{n+2 r}(X ; Q)=\sum_{i+j=n} \tilde{H}^{i}(X) \otimes \pi_{-j}(\mathbf{B U}, \mathbf{T U}) \rightarrow b^{n}(X ; \mathbf{B U}, \mathbf{T U})
$$

by our calculation of the groups $\pi_{n}(\mathrm{BU}, \mathrm{TU})$.

In more detail we state the following without proof. 
Coroll ary 4.2. (i) For $n=2 k<0, b^{n}(X ; \mathbf{B U}, \mathbf{T U}) \cong \Sigma \tilde{H}^{\text {even }}(X ; Q)$.

(ii) For $n=2 k+1<0, b^{n}(X ; \mathbf{B U}, \mathbf{T U}) \cong \Sigma \widetilde{H}^{\text {odd }}(X ; Q)$.

(iii) For $n>\operatorname{dim} X-2, b^{n}(X ; \mathbf{B U}, \mathbf{T U})=0$.

(iv) For $0 \leq n=2 k \leq \operatorname{dim} X-2, b^{n}\left(X ; \mathbf{B U}\right.$, TU) $=\Sigma_{r>k} \tilde{H}^{2 r}(X ; Q)$.

(v) For $1 \leq n=2 k+1 \leq \operatorname{dim} X-2, b^{n}(X ; \mathbf{B U}, \mathbf{T U})=\Sigma_{r>k} \widetilde{H}^{2 r+1}(X ; Q)$.

We next analyze the natural transformations $j_{*}: \widetilde{K}^{n} \rightarrow \Sigma_{r>0} \widetilde{H}^{n+2 r}(; Q)$.

Proposition 4.3. The natural transformation

$$
\widetilde{K}^{n} \stackrel{j_{*}}{\longrightarrow} b^{n}(; \mathbf{B U}, \mathbf{T U}) \cong \sum_{r>0} \widetilde{H}^{n+2 r}(; Q)
$$

is the truncated (from below) Chern character

$$
\mathrm{ch}^{\prime}=\sum_{r>0} \mathrm{ch}_{n+2 r}: \widetilde{K}^{n} \rightarrow \sum_{r>0} \widetilde{H}^{n+2 r}(; Q) .
$$

Proof. First recall from the remarks following Theorem 3.1, that $j_{*}: \pi_{2 s}$ (BU) $\rightarrow \pi_{2 s}(\mathbf{B U}, \mathbf{T U})$ is the inclusion $Z \subset Q$ for $s>0$, so $j_{*} \otimes 1: \pi_{2 s}(\mathbf{B U}) \otimes Q \rightarrow$ $\pi_{2 s}(\mathbf{B U}, \mathbf{T U}) \otimes Q \cong \pi_{2 s}(\mathbf{B U}, \mathbf{T U})$ is an isomorphism for $s>0$. Moreover $j_{*} \otimes 1$ is trivial in all other dimensions because $\pi_{r}(\mathbf{B U}, \mathbf{T U})$ is trivial then. Consider the commutative diagram

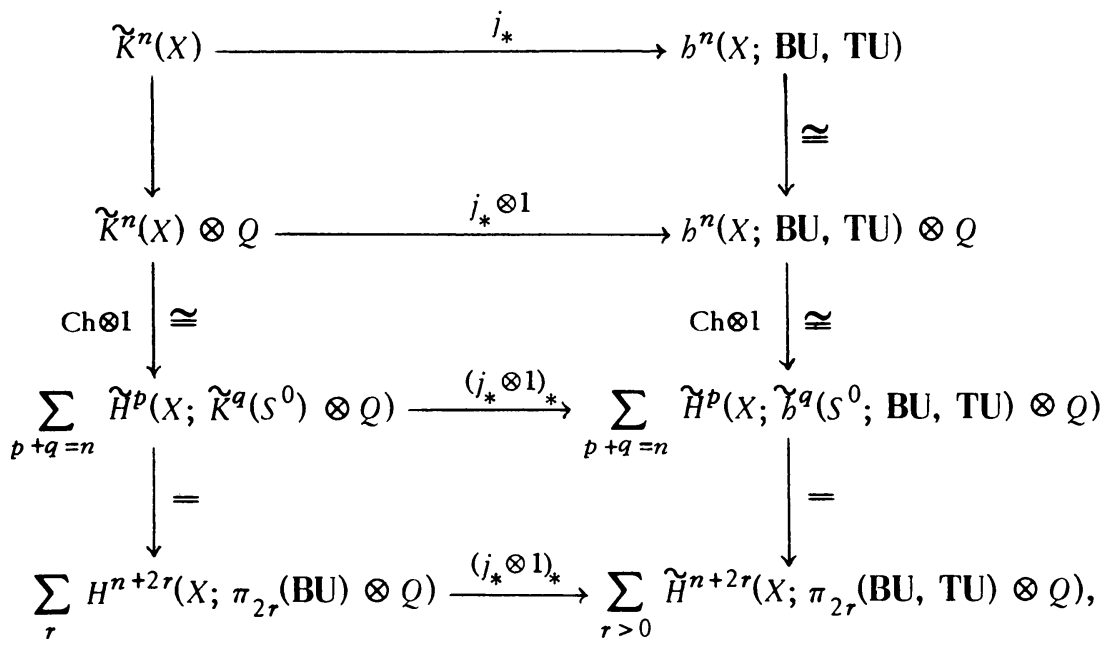

where $\mathrm{Ch}$ is the generalized Chern character of Dyer [6, p. 29]. By definition, the Chern character $\mathrm{ch}=\Sigma_{r} \mathrm{ch}_{n+2 r}$ is the vertical composite on the left, while the vertical composite on the right is the inverse of the equivalence of Proposition 4.1. Clearly the map $\left(j_{*} \otimes 1\right)_{*}$ of the bottom line is just projection on the summands for $r>0$ by the remarks above. Thus $(\mathrm{Ch} \otimes 1) \cdot j_{*}=\left(j_{*} \otimes 1\right)_{*} \mathrm{ch}=$ $\Sigma_{r>0} \mathrm{ch}_{n+2 r}=\mathrm{ch}^{\prime}$. 
Coroll ary 4.4. For $n<0$, the map $j_{*}$ is the Chern character, while for $\left.n\right\rangle$ $\operatorname{dim} X-2, j_{*}$ is trivial.

Let $T^{n}(X)=b^{n}(X ; \mathrm{TU})$. By the two previous propositions, the exact cohomology sequence may be written

$$
\cdots \rightarrow T^{n}(X) \stackrel{i_{*}}{\longrightarrow} \widetilde{K}^{n}(X) \stackrel{\mathrm{ch}^{\prime}}{\longrightarrow} \sum_{r>0} \widetilde{H}^{n+2 r}(X ; Q) \stackrel{\beta}{\longrightarrow} T^{n+1}(X) \rightarrow \cdots,
$$

where $\mathrm{ch}^{\prime}$ is the truncated Chern character.

Let $\widetilde{T}^{n}(X)=\operatorname{Ker} \mathrm{ch}^{\prime}=\operatorname{Im} i_{*} \subset \widetilde{K}^{n}(X)$, and let $D^{n-1}(X)=\operatorname{Im} \beta=\operatorname{Ker} i_{*} \subset T^{n}(X)$. For any abelian group $G$, let tors $G$ denote the torsion subgroup of $G$. The following proposition describes the structure of $T^{n}(X)$.

Proposition 4.5. (i) For each $n, T^{n}(X) \cong D^{n-1}(X) \oplus \widetilde{T}^{n}(X)$.

(ii) For each $n, D^{n}(X) \cong \Sigma_{r>0} \tilde{H}^{n+2 r}(X) \otimes Q / Z$.

(iii) For $n<0, \widetilde{T}^{n}(X)=\operatorname{tors} \widetilde{K}^{n}(X)$.

(iv) For $0 \leq n \leq \operatorname{dim} X-2, \widetilde{T}^{n}(X) \cong \operatorname{tors} \widetilde{K}^{n}(X) \oplus \Sigma_{r \leq 0} \tilde{H}^{n+2 r}(X) /$ tors $\widetilde{H}^{n+2 r}(X)$.

(v) For $\operatorname{dim} X-2<n, \widetilde{T}^{n}(X)=\widetilde{K}^{n}(X)$.

Proof. (i) Since $\Sigma_{r>0} \widetilde{H}^{n-1+2 r}(X ; Q)$ is divisible, $D^{n-1}(X)=\operatorname{Im} \beta$ is divisible. Now $0 \rightarrow D^{n-1}(X) \rightarrow T^{n}(X) \rightarrow T^{n}(X) / D^{n-1}(X) \rightarrow 0$ is exact, so $T^{n}(X) \cong$ $D^{n-1}(X) \oplus T^{n}(X) / D^{n-1}(X)$. But $i_{*}$ induces an isomorphism $T^{n}(X) / D^{n-1}(X) \rightarrow$ $\widetilde{T}^{n}(X)$ which yields part (i). Note that $\widetilde{T}^{n}(X) \subset \widetilde{K}^{n}(X)$ is finitely generated, so $D^{n-1}(X)$ is precisely the divisible subgroup of $T^{n}(X)$.

(ii) Since ch $\otimes 1: \widetilde{K}^{n}(X) \otimes Q \rightarrow \Sigma_{r} \widetilde{H}^{n+2 r}(X ; Q) \cong \Sigma_{r} \widetilde{H}^{n+2 r}(X) \otimes Q$ is an isomorphism,

$$
\operatorname{Im} \mathrm{ch} \cong \sum_{r} \widetilde{H}^{n+2 r}(X) / \text { tors } \widetilde{H}^{n+2 r}(X) \subset \sum_{r} \widetilde{H}^{n+2 r}(X) \otimes Q .
$$

From the commutative diagram

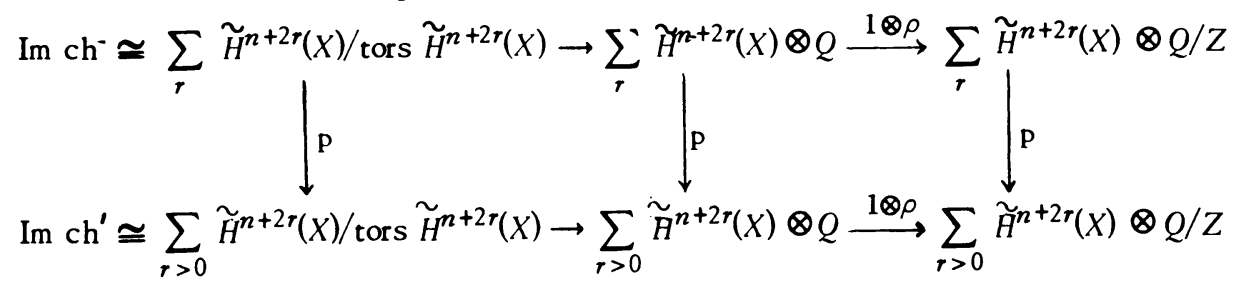

where $\rho: Q \rightarrow Q / Z$ is the usual projection and $p$ is a projection on a direct summand, we obtain $D^{n}(X)=\operatorname{Im} \beta \cong \Sigma_{r>0} \tilde{H}^{n+2 r}(X) \otimes Q / Z$.

(iii) For $n<0, \widetilde{T}^{n}(X)=\operatorname{Ker} \mathrm{ch}^{\prime}=\operatorname{Ker} \mathrm{ch}=\operatorname{tors} \widetilde{K}^{n}(X)$, by Corollary 4.4.

(iv) For $0 \leq n<\operatorname{dim} X-2$, write $c h=\mathrm{ch}^{\prime \prime}+\mathrm{ch}^{\prime}$, where $\mathrm{ch}^{\prime \prime}=\sum_{r \leq 0} \mathrm{ch}_{n}+2 r$ is the Chern character truncated from above. Then $\mathrm{ch} \mid \widetilde{T}^{n}(X)=\operatorname{ch}^{\prime \prime}: \stackrel{r}{T^{n}}(X) \stackrel{n+2 r}{\rightarrow}$ $\operatorname{Im~ch"} \cong \Sigma_{r \leq 0} \tilde{H}^{n+2 r}(X) /$ tors $\tilde{H}^{n+2 r}(X)$ is an epimorphism, so $\tilde{T}^{n}(X) \cong \operatorname{Ker}$ ch $\oplus$ $\operatorname{Im~ch"~} \cong$ tors $\widetilde{K}^{n}(X) \oplus \Sigma_{r \leq 0} \widetilde{H}^{n+2 r}(X) /$ tors $\tilde{H}^{n+2 r}(X)$. 
(v) For $\operatorname{dim} X-2<n, \mathrm{ch}^{\prime}=0$, so $\widetilde{T}^{n}(X)=\widetilde{K}^{n}(X)$ by Corollary 4.4 .

Remarks. Several of the isomorphisms used in the proof of this last proposition are not natural isomorphisms. For example the map

$$
\beta: \sum_{r>0} \widetilde{H}^{n+2 r}(X) \otimes Q \rightarrow D^{n}(X) \cong \sum_{r>0} \widetilde{H}^{n+2 r}(X) \otimes Q / Z
$$

is not reduction $\bmod Z$, or even of the form $1 \otimes \phi$ for some map $\phi: Q \rightarrow Q / Z$. An easy calculation for $X=C P^{2}$ shows this. A similar calculation for the map $f: C P^{2} \rightarrow S^{4}$ shows that $f^{*}: D^{0}\left(S^{4}\right) \rightarrow D^{0}\left(C P^{2}\right)$ is not the map $f^{*} \otimes 1: \widetilde{H}^{4}\left(S^{4}\right) \otimes Q / Z$ $\rightarrow \tilde{H}^{4}\left(C P^{n}\right) \otimes O / Z$.

If $f: Y \rightarrow X$, then $f^{*}=T^{n+1}(f): D^{n}(X) \rightarrow D^{n}(Y)$, since $D^{n}(X)$ is divisible, the homomorphic image of a divisible group is divisible, and $D^{n}(Y)$ is precisely the divisible subgroup of $T^{n+1}(Y)$. On the other hand, the splitting $T^{n+1}(X) \cong$ $D^{n}(X) \oplus \widetilde{T}^{n+1}(X)$ is not natural as can be seen by using the cofibration sequence $R P^{2 n-1} \stackrel{i}{\rightarrow} R P^{2 n} \rightarrow S^{2 n}$. We have a commutative diagram

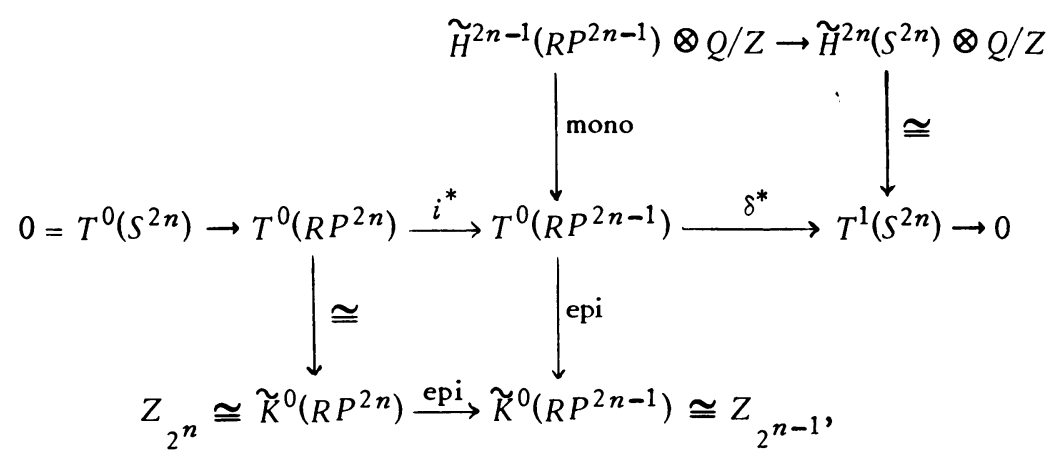

which implies the $T$ cohomology exact sequence in this dimension reduces to

$$
0 \rightarrow Z_{2^{n}} \stackrel{i^{*}}{\longrightarrow} Z_{2^{n-1}} \oplus Q / Z \stackrel{\delta^{*}}{\longrightarrow} Q / Z \rightarrow 0 .
$$

Clearly, if $g$ is a generator of $Z_{2 n}, i^{*}(g)$ must have a component in $Q / Z$ for $i^{*}$ to be a monomorphism.

Elementary properties of $\widetilde{T}^{n}$ and $D^{n}$ which follow easily from this proposition are as follows.

Corollary 4.6. If $X$ is rationally k-connected (i.e. $\pi_{i}(X) \otimes Q=0$ for $0 \leq i$ $\leq k)$, then $\widetilde{T}^{n}(X)=$ tors $\widetilde{K}^{n}(X)$ for $n \leq k$.

Corollary 4.7. Let $X$ be a complex such that $\Sigma_{r} \tilde{H}^{2 r+1}(X)=0$. Then

(i) $T^{2 r}(X) \cong \widetilde{T}^{2 r}(X)$

(ii) $T^{2 r+1}(X)=D^{2 r}(X)$.

The following proposition describes the exact $T$ cohomology sequence in an important special case. 
Proposition 4.8. Let $X$ be a complex sucb that $X^{2 n+1}=X^{2 n}$ and let $A \subset X$ be a subcomplex. There is an exact sequence

$$
\begin{aligned}
0 \rightarrow \tilde{T}^{2 r}(X, A) \stackrel{j^{*}}{\longrightarrow} \tilde{T}^{2 r}(X) \stackrel{i^{*}}{\longrightarrow} \tilde{T}^{2 r}(A) \stackrel{\delta^{*}}{\longrightarrow} D^{2 r}(X, A) \\
\stackrel{j^{*}}{\longrightarrow} D^{2 r}(X) \stackrel{i^{*}}{\longrightarrow} D^{2 r}(A) \rightarrow 0 .
\end{aligned}
$$

Proof. Except for the 0 groups on the end $s$ this is just a portion of the exact $T$-cohomology sequence of the pair $(X, A)$. To prove exactness at the ends, we need to prove $\delta^{*}: T^{2 s-1}(A) \rightarrow T^{2 s}(X, A)$ is trivial. But $T^{2 s-1}(A)=D^{2 s}(A) \cong$ $\Sigma_{t>0} \tilde{H}^{2(s+t)}(A) \otimes Q / Z$ is a torsion group while $T^{2 s}(X, A) \cong \widetilde{T}^{2 s}(X, A) \cong$ $\sum_{t \leq 0} \tilde{H}^{2(s+t)}(X, A)$ is torsion free. Thus $\delta^{*}=0$.

We remark that this exact sequence is of interest for $0 \leq r \leq$ dimension $X$. For $r \leq 0$ it reduces to the exact sequence for $\widetilde{H}^{*}(X ; Q / Z)$, while for $r \geq \operatorname{dim} X$ it reduces to the exact sequence for $\widetilde{K}$.

The map $\delta^{*}: \tilde{T}^{2 r}(A) \rightarrow D^{2 r}(X, A)$ is usually not trivial and carries considerable information about $X$. For example, by using the pairs $\left(C P^{n-1} / C P^{n-k-1}\right.$, $\left.C P^{n-2} / C P^{n-k-1}\right)$ one obtains divisibility properties of the James numbers $W\{n, k\}$. This will be the topic of a paper in preparation.

5. A filtration of $\widetilde{K}(X)$. If we now identify $\widetilde{K}^{2 r}(X)$ with $\widetilde{K}(X)$ and $\widetilde{K}^{2 r-1}(X)$ with $\widetilde{K}^{-1}(X)$, we obtain filtrations

$$
\begin{aligned}
\text { tors } \widetilde{K}(X) & =\widetilde{T}^{-2}(X) \subset \widetilde{T}^{0}(X) \subset \ldots \subset \widetilde{K}(X), \\
\text { tors } \widetilde{K}^{-1}(X) & =\widetilde{T}^{-1}(X) \subset \widetilde{T}^{1}(X) \subset \ldots \subset \widetilde{K}^{-1}(X) .
\end{aligned}
$$

Proposition 5.1. For $n \geq 0$ there is a monomorphism $\overline{\mathrm{ch}}_{n}: \widetilde{T}^{n}(X) / \tilde{T}^{n-2}(X) \rightarrow$ $\widetilde{H}^{n}(X ; Q)$ induced by the Chern character.

Proof. The diagram

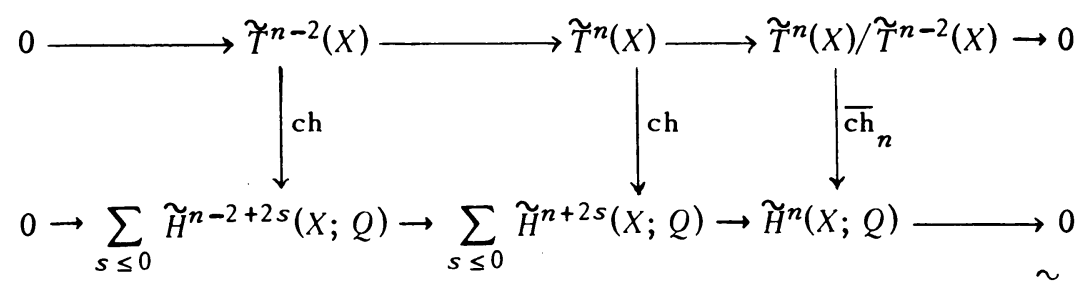

is commutative with exact rows, where $\overline{c h}_{n}$ is induced by ch. Since $\operatorname{ch}\left(\tilde{T}^{n-2}(X)\right)$ $=\operatorname{ch}\left(\widetilde{T}^{n}(X)\right) \cap \Sigma_{s \leq 0} \tilde{H}^{n-2+2 s}(X ; Q), \overline{c h}_{n}$ is a monomorphism.

Corollary 5.2. For $n \geq 0$, the group $\widetilde{T}^{n}(X) / \widetilde{T}^{n-2}(X)$ is free abelian.

Since $\widetilde{K}$ and $\widetilde{K}^{-1}$ are not naturally graded functors, the Chern character ch: $\widetilde{K} \rightarrow \Sigma_{r} \widetilde{H}^{2 r}(; Q)$ is not usually studied with respect to the graded structure available in rational cohomology. The filtrations above may be used to build a graded structure in $\widetilde{K}$ related to the Chern character. 
Throughout the remainder of this section we will concentrate on the functor $\widetilde{K}$, since $\widetilde{\widetilde{K}}^{-1}(X)=\widetilde{K}(S X)$.

Theorem 5.3. There is a natural isomorpbism $\phi$ sucb that the following diagram commutes:

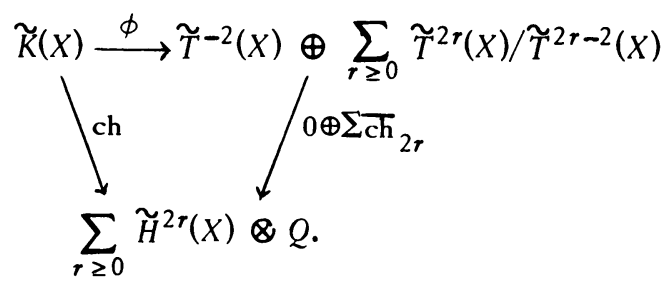

Proof. One proves inductively the existence of natural isomorphisms and commutative diagrams

$$
\begin{aligned}
& \tilde{T}^{2 s}(X) \stackrel{\phi^{2 s}}{\longrightarrow} \tilde{T}^{-2}(X) \underset{\sum_{r \leq s}}{\oplus} \tilde{T}^{2 r}(X) / \tilde{T}^{2 r-2}(X) \\
& \sum_{r \leq s} \tilde{H}^{2 r}(X) \otimes Q \text {. }
\end{aligned}
$$

Since $\tilde{T}^{2 s}(X)=\widetilde{K}(X)$ for $s$ large enough the theorem follows.

Corollary 5.4. There is a basis $\xi_{1}, \cdots, \xi_{N}$ for $\tilde{K}(X) /$ tors $\tilde{K}(X)$ such that $\xi_{1}, \ldots, \xi_{n_{s}}$ is a basis for $\tilde{T}^{2 s}(X) / \tilde{T}^{-2}(X)$. Moreover one can select a basis for $\Sigma_{r} \tilde{H}^{2 r}(X ; Q)$ so that with respect to these bases, the matrix of ch bas the form

$$
\mathrm{ch}=\left(\begin{array}{cccc}
C_{00} & C_{01} & C_{02} & C_{0 m} \\
0 & C_{11} & C_{12} & C_{1 m} \\
\cdot & \cdot & \cdot & \cdot \\
\cdot & \cdot & \cdot & \cdot \\
\cdot & \cdot & \cdot & \cdot \\
0 & 0 & 0 & C_{m m}
\end{array}\right)
$$

where $C_{i j}$ is an $\left(n_{i}-n_{i-1}\right) \times\left(n_{j}-n_{j-1}\right)$ matrix over $Q$ and $C_{i i}$ is nonsingular.

Proof. Select bases $\bar{\xi}_{i}, n_{r-1}<i \leq \sim_{\sim}{ }_{r}$ for $T_{\sim}^{2 r}(X) / \widetilde{T}_{\sim}^{2 r-2}(X)$ and choose $\xi_{i}, n_{r-1}<i \leq n_{r}$, to be a preimage in $\tilde{T}^{2 r}(X) / \tilde{T}^{-2}(X) \subset \tilde{K}(X) /$ tors $\tilde{K}(X)$. Then $\xi_{1}, \cdots, \xi_{N}$ will be a basis of the type stated.

Select a basis $x_{\sim_{1}}, \cdots, x_{N}$ for $\Sigma_{r} \tilde{H}^{2 r}(X) /$ tors $\tilde{H}^{2 r}(X)$ such that $x_{1}, \cdots, x_{n_{s}}$ is a basis for $\Sigma_{r \leq s} \tilde{H}^{2 r}(X) /$ tors $\tilde{H}^{2 r}(X)$. Then $x_{1} \otimes 1, \cdots, x_{N} \otimes 1$ is a basis for $\Sigma_{r} \widetilde{H}^{2 r}(X) \otimes Q$. Since ch: $\widetilde{T}^{2 s}(X) \rightarrow \Sigma_{r \leq \Sigma} \widetilde{H}^{2 r}(X) \otimes Q$, the matrix has the form stated. Since ch is a monomorphism on $\widetilde{K}(X)$ /tors $\widetilde{K}(X)$ the diagonal blocks are nonsingular. 
Examples 5.5. Recall that $\tilde{K}\left(C P^{n}\right)=Z[\xi] /\left(\xi^{n+1}\right)$ where $\xi=\eta-1$ and $\eta$ is the canonical line bundle over $C P^{n}$.

For $C P^{2}$ we have $\widetilde{T}^{2 r}\left(C P^{2}\right)=0$ for $r \leq 0$ and $\widetilde{T}^{2 r}\left(C P^{2}\right)=\widetilde{K}\left(C P^{2}\right)$ for $r \geq 2$. If we choose $\theta_{1}=2 \xi-\xi^{2}, \theta_{2}=\xi$, then $\theta_{1}, \theta_{2}$ is a basis for $\widetilde{K}\left(C P^{2}\right)$ such that $\theta_{1}$ is a basis for $\tilde{T}^{2}(X)$.

For $C P^{3}$ we have $\widetilde{T}^{2 r}\left(C P^{3}\right)=0$ for $r \leq 0$ and $\widetilde{T}^{2 r}\left(C P^{3}\right)=\widetilde{K}\left(C P^{3}\right)$ for $r \geq 3$. If we choose $\theta_{1}=6 \xi-3 \xi^{2}+2 \xi^{3}, \theta_{2}=\xi^{2}-\xi^{3}, \theta_{3}=5 \xi-\xi^{3}$, then $\theta_{1}, \theta_{2}, \theta_{3}$ is a basis for $\widetilde{K}\left(C P^{3}\right)$ such that $\theta_{1}$ is a basis for $\widetilde{T}^{2}\left(C P^{3}\right)$, and $\theta_{1}, \theta_{2}$ is a basis for $\widetilde{T}^{4}\left(C P^{3}\right)$.

Lemm a 5.6. Suppose we have a commutative diagram with exact rows and columns

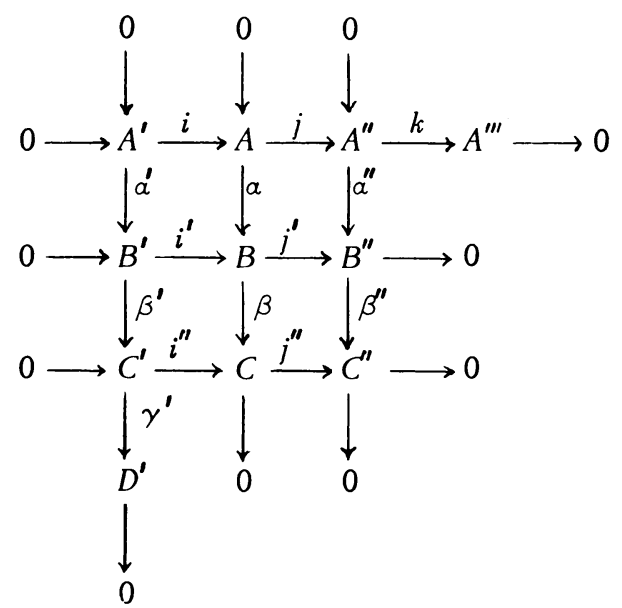

There is an isomorphism $\phi: D^{\prime} \rightarrow A^{\prime \prime \prime}$.

Proof. Define $\phi=k \alpha^{\prime \prime-1} j^{\prime} \beta^{-1} i^{\prime \prime} \gamma^{\prime-1}$. Using standard diagram chasing techniques one proves $\phi$ is well defined, a homomorphism, and has inverse $\phi^{-1}=$ $\gamma^{\prime} i^{\prime \prime-1} \beta j^{\prime-1} \alpha^{\prime \prime} k^{-1}$.

Let $f: X \rightarrow Y$ and suppose $f^{*}: \widetilde{K}(Y) \rightarrow \widetilde{K}(X)$ is an epimorphism. If $\widetilde{K}(X)$ is a free abelian group we know $\widetilde{K}(Y) \cong \operatorname{Ker} f^{*} \oplus \tilde{K}(X)$. Although we may certainly split $\Sigma_{r} \widetilde{H}^{2 r}(Y) \otimes Q \cong \operatorname{ker} f^{*} \oplus \Sigma_{r} \widetilde{H}^{2 r}(X) \otimes Q$ one cannot usually assert that $\mathrm{ch}_{Y}=\left(\mathrm{ch}_{Y} \mid \operatorname{Ker} f^{*}\right) \oplus \mathrm{ch}_{X}$. The following theorem is a criterion for such a splitting of ch.

Theorem 5.7. Let $f: X \rightarrow Y$ be such that $f^{*}: \tilde{K}(Y) \rightarrow \widetilde{K}(X)$ is an epimorphism, where $\tilde{K}(X)$ is free abelian. Then the Chern character splits ch $=\mathrm{ch} \mid \operatorname{Ker} f^{*} \oplus \mathrm{ch}$ : $\operatorname{Ker} f^{*} \oplus \tilde{K}(X) \rightarrow \operatorname{Ker} f^{*} \oplus \Sigma_{r} \tilde{H}^{2 r}(X) \otimes Q$ if and only if $f^{*}: \tilde{T}^{i s}(Y) \rightarrow \tilde{T}^{2 s}(X)$ is an epimorphism for all $s$. 
Proof. Let $G=\operatorname{Ker}\left(f^{*}: \tilde{K}(Y) \rightarrow \widetilde{K}(X)\right)$, and define the groups $G^{2 s}$ and $H^{2 s}$ for $s \geq-1$ so that the following diagram is commutative with exact rows and columns

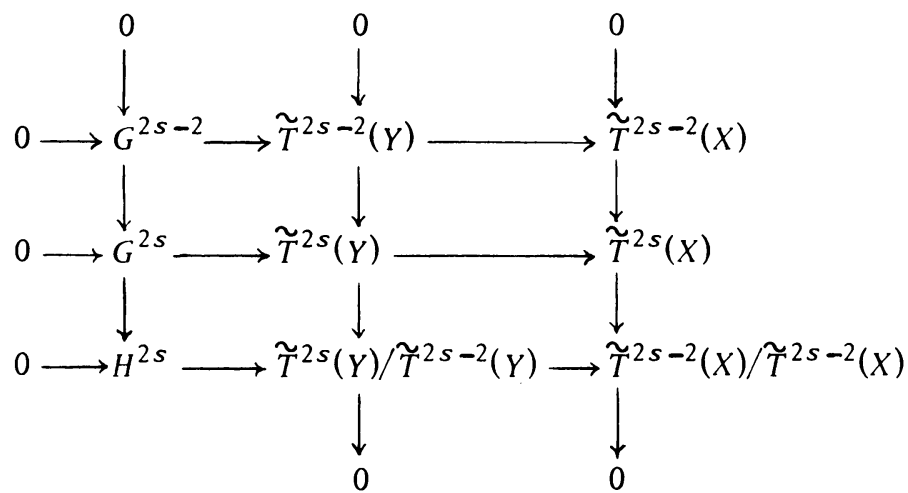

Since $G^{2 s}=G \cap \widetilde{T}^{2 s}(Y)$ it follows that $G^{2 s} / G^{2 s-2} \subset H^{2 s} \subset \widetilde{T}^{2 s}(Y) / \widetilde{T}^{2 s-2}(Y)$ and hence is free abelian. An easy inductive proof on the filtration $G^{-2} \subset G^{0} \subset$ $G^{2} \subset \ldots \subset G$ establishes a commutative diagram and isomorphisms

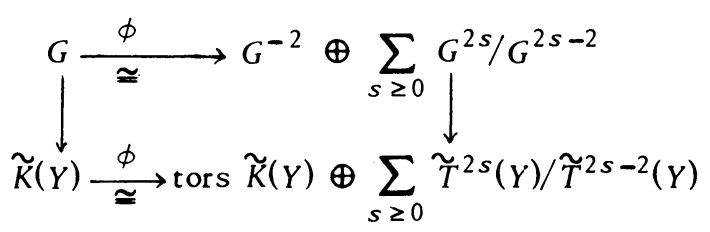

On the other hand, from the $3 \times 3$ diagram above and Lemma 5.6, it follows that if $\tilde{T}^{2 s}(Y) \rightarrow \widetilde{T}^{2 s}(X)$ is an epimorphism, then $G^{2 s} \rightarrow H^{2 s}$ is an epimorphism (i.e. $\left.G^{2 s} / G^{2 s-2}=H^{2 s}\right)$ if and only if $\tilde{T}^{2 s-2}(Y) \rightarrow \widetilde{T}^{2 s-2}(X)$ is an epimorphism. Thus by descending induction on $s$ (we start with $f^{*}: \tilde{K}(Y) \rightarrow \tilde{K}(X)$ is an epimorphism) we obtain the fact that $G-\cap \widetilde{T}^{2 s}(X) / \widetilde{T}^{2 s-2}(X)=G^{2 s} / G^{2 s-2}$ if and only if $f^{*}$ : $\tilde{T}^{2 s}(Y) \rightarrow \widetilde{T}^{2 s}(X)$ is an epimorphism for all $s$. Since

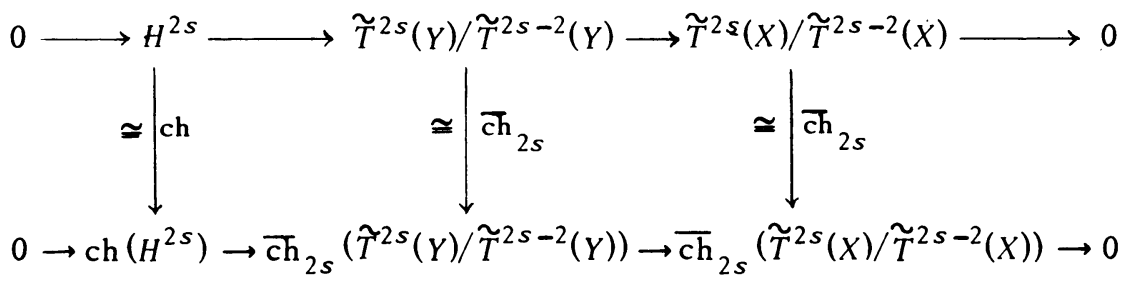

is a commutative diagram of free abelian groups with exact rows, it follows that

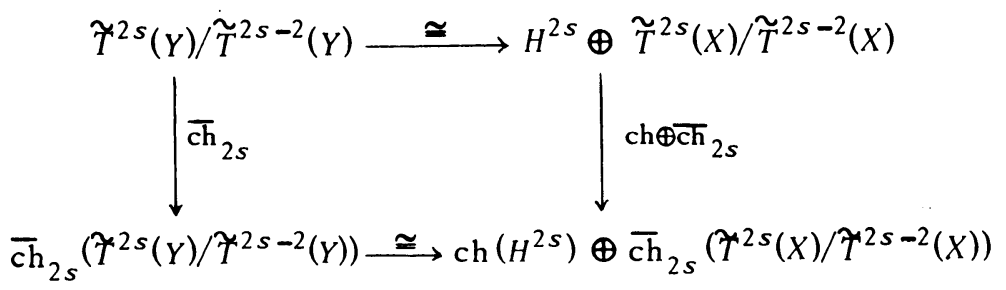


commutes and $\mathrm{ch}=\overline{\mathrm{ch}}_{2 s} \mid G^{2 s} / G^{2 s-2}$ if and only if $H^{2 s}=G^{2 s} / G^{2 s-2}$. Thus by Theorem 5.3 we have a commutative diagram

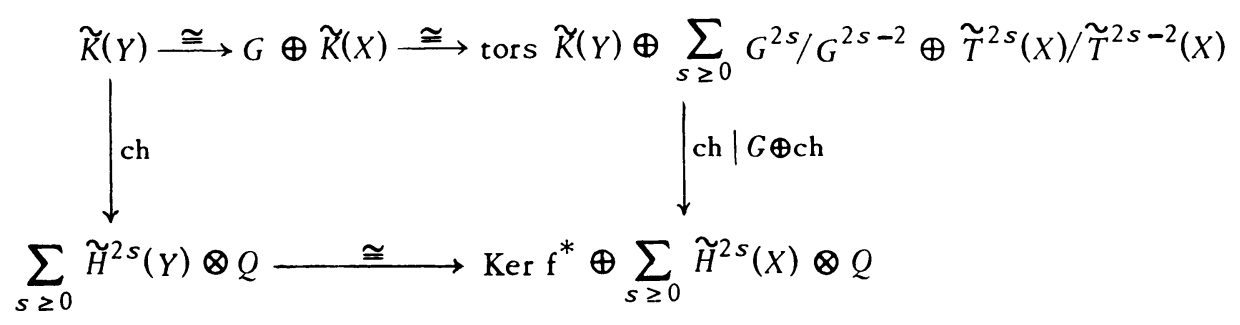

if and only if $f^{*}: \tilde{T}^{2 s}(Y) \rightarrow \widetilde{T}^{2 s}(X)$ is an epimorphism.

We remark that the assumption that $\tilde{K}(X)$ is torsion free is only a notational convenience in the theorem above. If $\widetilde{K}(X)$ has torsion, replace $\widetilde{K}(X)$ by $\tilde{K}(X) /$ tors $\tilde{K}(X)$ and $\tilde{T}^{2 s}(X)$ by $\tilde{T}^{2 s}(X) / \widetilde{T}^{-2}(X)$ throughout the theorem and its proof.

The following proposition shows that the filtration tors $\tilde{K}(X)=\widetilde{T}^{-2}(X) \subset$ $\tilde{T}^{0}(X) \subset \cdots \subset \tilde{K}(X)$ behaves nicely with respect to products.

Proposition 5.8. The internal product $\widetilde{K}(X) \otimes \widetilde{K}(X) \rightarrow \widetilde{K}(X)$ induces a pairing $\tilde{T}^{2 p}(X) \otimes \tilde{T}^{2 q}(X) \rightarrow \widetilde{T}^{2(p+q)}(X)$.

Proof. If $\xi \in \widetilde{T}^{2 p}(X)$ and $\eta \in \widetilde{T}^{2 q}(X)$, then $\operatorname{ch}(\xi \cdot \eta)=\operatorname{ch}(\xi) \operatorname{ch}(\eta) \in \sum_{r \leq 0} \widetilde{H}^{2(p+r)}(X ; Q) \sum_{s \leq 0} \widetilde{H}^{2(q+s)}(X ; Q) \subset \sum_{t \leq 0} \tilde{H}^{2(p+q+t)}(X ; Q)$. Thus $\xi \cdot \eta \in \widetilde{T}^{2(p+q)}(X)$.

We next investigate the effect of the Adams operations $\psi^{k}$ on the group $\tilde{T}^{r}(X)$.

Proposition 5.9. Let $\xi \in \widetilde{K}(X)$ and $k>0$. Then $\psi^{k}(\xi) \in \widetilde{T}^{2 n}(X)$ if and only if $\xi \in \widetilde{T}^{2 n}(X)$.

Proof. According to Adams [1, Theorem 5.1], for $\xi \in \tilde{K}(X)$,

$$
\operatorname{ch}\left(\psi^{k} \xi\right)=\sum_{r} \operatorname{ch}_{2(n+r)}\left(\psi^{k}(\xi)\right)=\sum_{r} k^{n+r} \operatorname{ch}_{2(n+r)}(\xi) \text {. }
$$

Thus $\operatorname{ch}_{2(n+r)}\left(\psi^{k} \xi\right)=0$ for $r>0$ if and only if $\operatorname{ch}_{2(n+r)}(\xi)=0$ for $r>0$. Equivalently $\psi^{k}(\xi) \in \widetilde{T}^{2 n}(X)$ if and only if $\xi \in \widetilde{T}^{2 n}(X)$.

Since $\psi^{k}: \widetilde{T}^{2 n}(X) \rightarrow \widetilde{T}^{2 n}(X)$ for $k>0$ we have induced maps $\bar{\psi}^{k}$ : $\widetilde{T}^{2 n}(X) / \widetilde{T}^{2 n-2}(X) \rightarrow \widetilde{T}^{2 n}(X) / \widetilde{T}^{2 n-2}(X)$.

Corollary 5.10. For $\bar{\xi} \in \widetilde{T}^{2 n}(X) / \widetilde{T}^{2 n-2}(X), \bar{\psi}^{k}(\bar{\xi})=k^{n} \bar{\xi}$.

It is of course well known that on the torsion-free part of $\tilde{K}(X)$ the operations $\psi^{k}$ are determined by the Chern character. With the aid of Corollary 5.4, we are able to determine a formula for the $\psi^{k}$.

Theorem 5.11. Let $\xi_{1}, \cdots, \xi_{N}$ be a basis for $\tilde{K}(X) / \tilde{T}^{-2}(X)$ such that 
$\xi_{1}, \cdots, \xi_{n_{r}}$ is a basis for $\widetilde{T}^{2 r}(X) / T^{-2}(X)$. If $n_{r}<i \leq n_{r+1}$ and $j \leq n_{r}$ there exist polynomials $P_{i j}(y)=p_{i j}^{0}+p_{i j}^{1} y+\cdots+p_{i j}^{r+1} y^{r+1}$ sucb that $\psi^{k} \xi_{i}=k^{r+1} \xi_{i}+$ $\sum_{j=1}^{n_{r}} P_{i j}(k) \xi_{j}$.

We remark that even though the polynomials $P_{i j}(y)$ have rational coefficients, they assume integral values for each positive integer.

Proof. By Corollary 5.10, $\psi^{k}\left(\xi_{i}\right)-k^{r+1} \xi_{i} \in \widetilde{T}^{2 r}(X)$ for $n_{r}<i \leq n_{r+1}$, so the matrix of $\psi^{k}$ may be written $\psi^{k}=I(k)+P(k)$, where

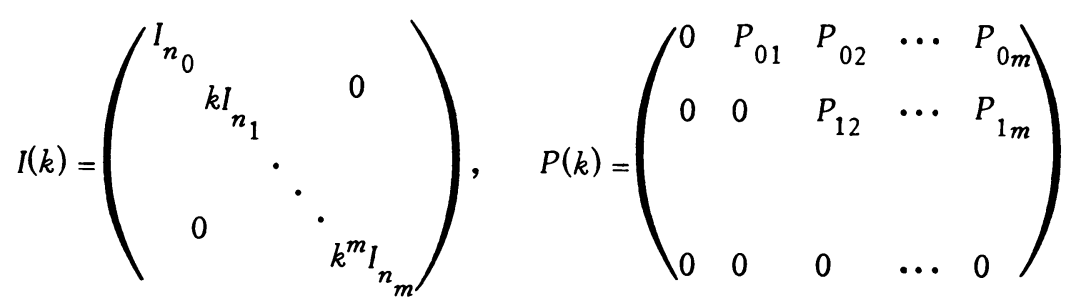

on the free abelian group $\tilde{K}(X) / \tilde{T}^{-2}(X)$. Now $\operatorname{ch} \psi^{k}=\operatorname{ch}(I(k)+P(k))$, but according to Adams [1, Theorem 5.1], ch $\psi^{k}=I(k)$ ch. Thus $\operatorname{ch}(I(k)+P(k))=I(k) \mathrm{ch}$ or $P(k)=\mathrm{ch}^{-1} I(k) \mathrm{ch}-I(k)$. The polynomials $P_{i j}(y)$ are entries in the matrix $P(y)=$ $\mathrm{ch}^{-1} I(y) \mathrm{ch}-I(y)$.

Examples 5.12. In $\widetilde{K}\left(C P^{2}\right)$ with the basis selected above $\psi^{k}\left(\theta_{1}\right)=k \theta_{1}$, $\psi^{k}\left(\theta_{2}\right)=k^{2} \theta_{2}+1 / 2\left(k-k^{2}\right) \theta_{1}$.

In $\tilde{K}\left(C P^{3}\right)$ with the basis selected above $\psi^{k} \theta_{1}=k \theta_{1}, \psi^{k} \theta_{2}=k^{2} \theta_{2}, \psi^{k} \theta_{3}=$ $k^{3} \theta_{3}+5\left(k^{2}-k^{3}\right) \theta_{2} / 2+5\left(k-k^{3}\right) \theta_{1} / 6$.

6. On the cokernel of ch. If we identify $\widetilde{K}^{2 s}(X)$ with $\widetilde{K}(X)$ and $\widetilde{K}^{2 s-1}(X)$ with $\widetilde{K}^{-1}(X)$ then we have a commutative diagram

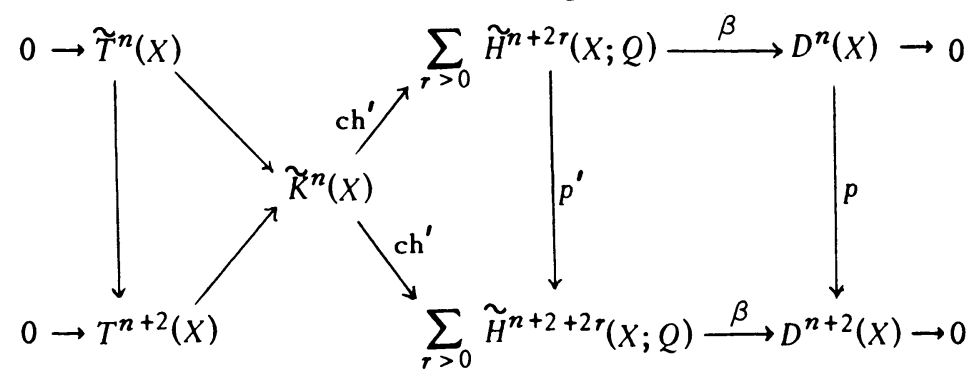

where $p^{\prime}$ and consequently $p: \Sigma_{r>0} \tilde{H}^{n+2 r}(X) \otimes Q / Z \rightarrow \Sigma_{r>0} \tilde{H}^{n+2+2 r}(X) \otimes Q / Z$ are projections on direct summands. We thus obtain cofiltrations

$$
\begin{aligned}
& \sum_{r} \widetilde{H}^{2 n+2 r}(X) \otimes Q / Z=D^{-2}(X) \rightarrow D^{0}(X) \rightarrow \cdots \rightarrow 0 \\
& \sum_{r} \widetilde{H}^{2 n-1+2 r}(X) \otimes Q / Z=D^{-1}(X) \rightarrow D^{1}(X) \rightarrow \cdots \rightarrow 0
\end{aligned}
$$


Observe that $\operatorname{ker}\left(p: D^{n}(X) \rightarrow D^{n+2}(X)\right)=\tilde{H}^{n+2}(X) \otimes Q / Z$.

We noted in $\$ 4$ that in general $\beta: \Sigma_{r>0} \tilde{H}^{n+2 r}(X) \otimes Q \rightarrow \Sigma_{r>0} \tilde{H}^{n+2 r}(X) \otimes Q / Z$ was not of the form $1 \otimes \rho$ for some reduction $\bmod Z \rho: Q \rightarrow Q / Z$. However for a restriction of $\beta$ we have an exact sequence

$$
\begin{aligned}
0 \rightarrow & \operatorname{Im} \operatorname{ch}_{s} \longrightarrow \tilde{H}^{s}(X) \otimes Q \stackrel{\beta}{\longrightarrow} \tilde{H}^{s}(X) \otimes \cdot Q / Z \rightarrow 0 \\
& \mathbb{R} \\
& \tilde{H}^{s}(X) / \text { tors } \tilde{H}^{s}(X),
\end{aligned}
$$

and by proper choice of the bases in $\operatorname{Im} c h$ and $\tilde{H}^{s}(X) /$ tors $\tilde{H}^{s}(X)$ we can assume the restriction $\beta$ is of the form $1 \otimes \rho$ for some reduction $\bmod Z \rho: Q \rightarrow Q / Z$.

The following theorem is dual to Theorem 5.7.

Theorem 6.1. Let $f: X \rightarrow Y$ be such that $f^{*}: \tilde{H}^{*}(Y) \rightarrow \tilde{H}^{*}(X)$ is a monomorphism, where $\tilde{H}^{*}(Y)$ is torsion free. Then the map $\beta: \Sigma_{s} \tilde{H}^{s}(X) \otimes Q \rightarrow$ $\Sigma_{s} \tilde{H}^{s}(X) \otimes Q / Z$ is a graded homomorphism with components of the form $1 \otimes \rho$ where $\rho$ is some reduction $\bmod Z, \rho: Q \rightarrow Q / Z$ if and only if $f^{*}: D^{n+2 r}(Y) \rightarrow$ $D^{n+2 r}(X)$ is a monomorphism for all $r$.

Proof. The proof is dual to that of Theorem 5.7 and will not be given. One first proves a dual version of Lemma 5.6.

7. The $e$-invariant. For any map $f: X \rightarrow Y$, let $f^{\prime *}: \tilde{T}^{n}(Y) \rightarrow \tilde{T}^{n}(X)$ be the restriction of $f_{K}^{*}: \tilde{K}^{*}(Y) \rightarrow \widetilde{K}^{*}(Y)$. Since the homomorphic image of a divisible subgroup is divisible, the restriction of $f_{T}^{*}:{\underset{\sim}{T}}^{n+1}(Y) \rightarrow T^{n+1}(X)$ yields a homomorphism $f^{\prime \prime *}: D^{n}(Y) \rightarrow D^{n}(X)$. We will identify $\widetilde{T}^{n}(X)$ with the direct summand of $T^{n}(X)$ isomorphic to it whenever necessary.

Now consider a cofibration sequence $A \stackrel{i}{\rightarrow} X \stackrel{j}{\rightarrow} X / A \stackrel{d}{\rightarrow} S A$ and the corresponding. $T$-cohomology exact sequence.

Proposition 7.1. Let $A \stackrel{i}{\rightarrow} X \stackrel{j}{\rightarrow} X / A$ be a cofibration such that $i_{K}^{*}: \tilde{K}^{*}(X)$ $\rightarrow \tilde{K}^{*}(A)$ is an epimorphism. Then for each $n$, the sequence

$$
\begin{aligned}
0 \rightarrow \widetilde{T}^{n}(X / A) \stackrel{j^{*}}{\longrightarrow} \tilde{T}^{n}(X) \stackrel{i^{*}}{\longrightarrow} \widetilde{T}^{n}(A) \stackrel{\delta^{*}}{\longrightarrow} D^{n}(X / A) \\
\stackrel{i^{\prime *}}{\longrightarrow} D^{n}(X) \stackrel{i^{\prime *}}{\longrightarrow} D^{n}(A) \rightarrow 0
\end{aligned}
$$

is exact.

Proof. Since $i_{K}^{*}: \tilde{K}(X) \rightarrow \tilde{K}(A)$ is an epimorphism, the cohomology map $i^{*}$ : $\tilde{H}^{*}(X ; Q) \rightarrow \widetilde{H}^{*}(A ; Q)$ is an epimorphism. Thus for each $n$ we have a commutative diagram 


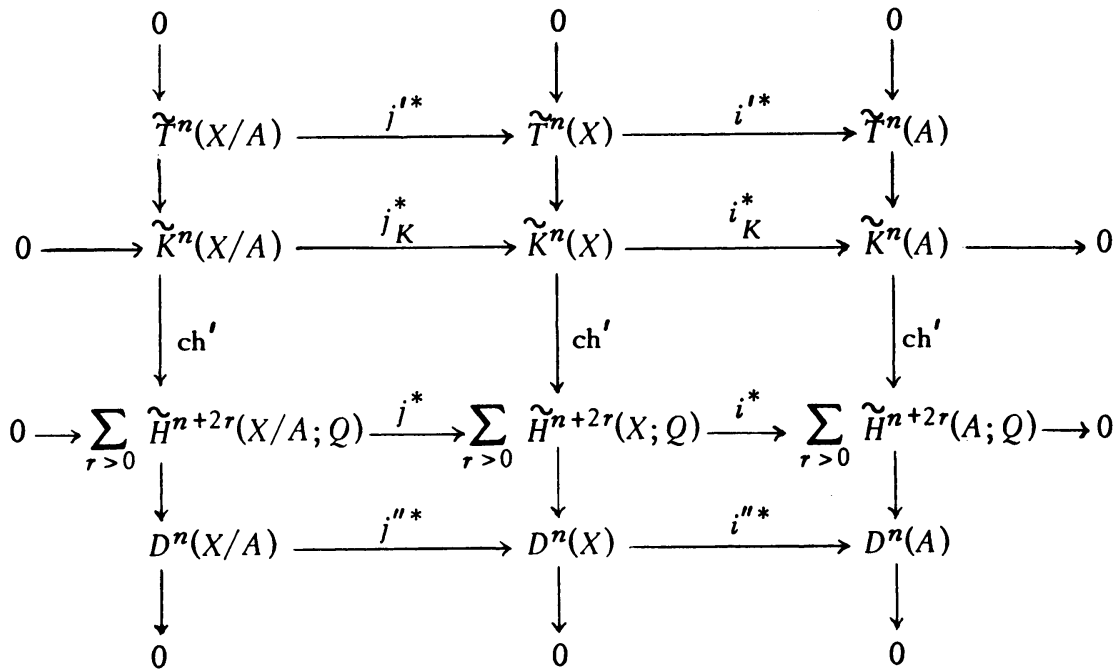

where the two center rows and all columns are exact. An elementary diagram chase establishes that

$$
0 \rightarrow \tilde{T}^{n}(X / A) \stackrel{j^{*}}{\longrightarrow} \tilde{T}^{n}(X) \stackrel{i^{*}}{\longrightarrow} \tilde{T}^{n}(A)
$$

and

$$
D^{n}(X / A) \stackrel{j^{\prime *}}{\longrightarrow} D^{n}(X) \stackrel{i^{\prime *}}{\longrightarrow} D^{n}(A) \rightarrow 0
$$

are both exact.

From the diagram

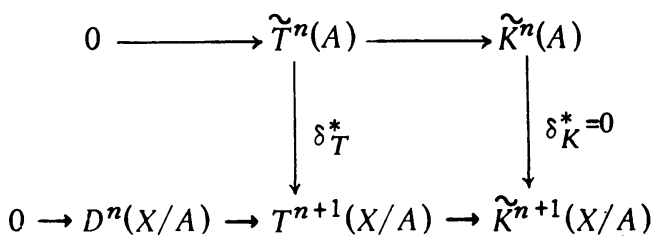

it follows that $\operatorname{Im}\left(\delta_{T}^{*} \mid \tilde{T}^{n}(A)\right) \subset D^{n}(X / A)$. From exactness of $D^{n-1}(X) \stackrel{i^{\prime \prime}}{\rightarrow} D^{n-1}(A)$ $\rightarrow 0$, it follows that $\delta_{T}^{*} \mid D^{n-1}(A)=0$ and $\delta_{T}^{*}\left(\tilde{T}^{n}(A)\right)=\operatorname{Ker}\left(D^{n}(X / A) \stackrel{j^{\prime \prime}}{\rightarrow} D^{n}(X)\right)$.

From the diagram

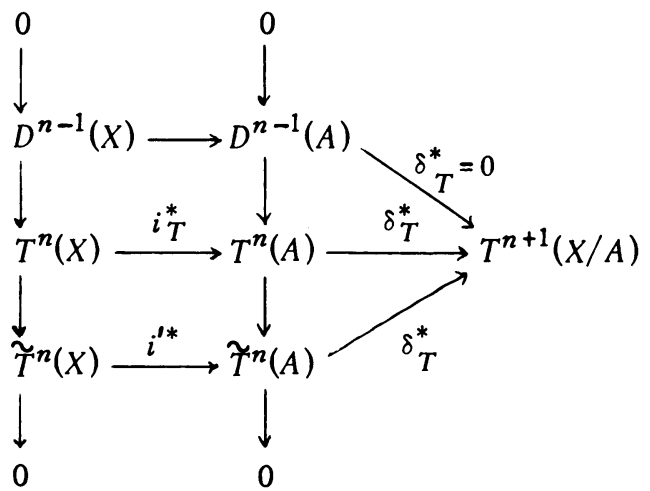


it follows that $\operatorname{Ker}\left(\delta_{T}^{*} \mid \tilde{T}^{n}(A)\right)=\operatorname{Im} i^{\prime *}$. We piece this together to obtain the exact sequence of the proposition.

Remark. Clearly an analogous proposition and proof is available if any of the other maps in the $K$-theory exact sequence is trivial. For example, if $i_{K}^{*}: \tilde{K}^{*}(X)$ $\rightarrow \widetilde{K}^{*}(A)$ is trivial, then

$$
0 \rightarrow \tilde{T}^{n-1}(A) \rightarrow \tilde{T}^{n}(X / A) \rightarrow \tilde{T}^{n}(X) \stackrel{i_{T}^{*}}{\longrightarrow} D^{n-1}(A)
$$

is exact, and if $j_{K}^{*}: \widetilde{K}^{*}(X / A) \rightarrow \widetilde{K}^{*}(X)$ is trivial, then

$$
\longrightarrow D^{n}(X / A) \rightarrow D^{n}(X) \rightarrow 0
$$

$$
0 \rightarrow \tilde{T}^{n-1}(X) \rightarrow \tilde{T}^{n-1}(A) \rightarrow \tilde{T}^{n}(X / A) \stackrel{j_{T}^{*}}{\longrightarrow} D^{n-1}(X)
$$

is exact.

$$
\longrightarrow D^{n-1}(A) \rightarrow D^{n}(X / A) \rightarrow 0
$$

An important special case for application is the following.

Corollary 7.2. Let $f: X \rightarrow Y$ be a map sucb that $f_{K}^{*}: \tilde{K}^{*}(Y) \rightarrow \tilde{K}^{*}(X)$ is trivial. Then for eacb integer $n$,

$$
\begin{aligned}
0 \rightarrow \tilde{T}^{n}(S X) \stackrel{j^{\prime *}}{\longrightarrow} \tilde{T}^{n}(Y \cup, C X) \stackrel{i^{\prime *}}{\longrightarrow} \tilde{T}^{n}(Y) \stackrel{\delta_{T}^{*}}{\longrightarrow} D^{n}(S X) \\
\stackrel{j^{\prime *}}{\longrightarrow} D^{n}(Y \cup, C X) \stackrel{i^{\prime *}}{\longrightarrow} D^{n}(Y) \rightarrow 0
\end{aligned}
$$

is exact.

Let $f: X \rightarrow Y$ be a map such that $f^{*}: \widetilde{K}^{*}(Y) \rightarrow \widetilde{K}^{*}(X)$ is trivial. Form the cofibration sequence $Y \stackrel{i}{\rightarrow} Y \cup, C X \stackrel{j}{\rightarrow} S X$ and define

$$
e^{n}(f)=\delta_{T}^{*}: \widetilde{T}^{n}(Y) \rightarrow D^{n}(S X) \cong \sum_{r>0} \widetilde{H}^{n+2 r}(S X) \otimes Q / Z .
$$

Consider the commutative diagram

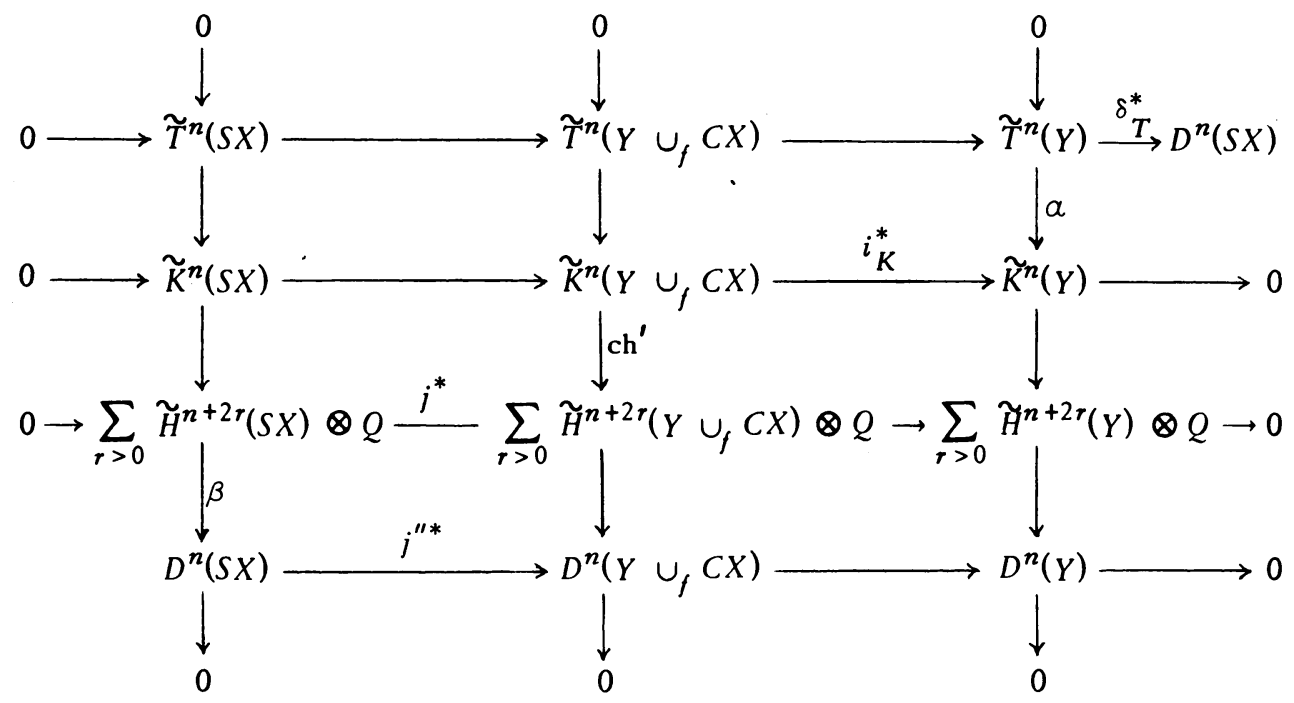


with exact rows and columns, and define $\lambda^{n}(f): \widetilde{T}^{n}(Y) \rightarrow D^{n}(S X)$ by $\lambda^{n}(f)=$ $\beta j^{*-1} \mathrm{ch}^{\prime} i_{K}^{*}{ }^{-1} \alpha$. A simple diagram chase establishes that $\lambda^{n}(f)$ is a well-defined homomorphism, and by Lemma 5.6, there is a homomorphism $\phi: \operatorname{Im} \delta_{T}^{*} \rightarrow D^{n}(S X)$ which is an isomorphism onto $\operatorname{Ker} i^{\prime *} \subset D^{n}(S X)$ and $\phi \delta_{T}^{*}=\lambda^{n}(f)$.

Thus we see that $e^{n}(f)$ is essentially $\lambda^{n}(f)$, i.e. $e^{n}(f)$ differs from $\lambda^{n}(f)$ by an automorphism on its image.

Let $q>n$ and let $f: S^{2 q-1} \rightarrow S^{2 n}$. Then $0 \rightarrow \widetilde{K}\left(S^{2 q}\right) \stackrel{j^{*}}{\rightarrow} \tilde{K}\left(S^{2 n} \cup, e^{2 q}\right) \stackrel{i^{*}}{\rightarrow}$ $\tilde{K}\left(S^{2 n}\right) \rightarrow 0$ is exact and the invariant $\lambda^{2 n}(f)$ takes the form

$$
\lambda^{2 n}(f): \widetilde{T}^{2 n}\left(S^{2 n}\right) \rightarrow D^{2 n}\left(S^{2 q}\right) \cong H^{2 q}\left(S^{2 q}\right) \otimes Q / Z \cong Q / Z
$$

Proposition 7.3. If $\xi \in \widetilde{T}^{2 n}\left(S^{2 n}\right) \cong \tilde{K}^{2 n}\left(S^{2 n}\right)$ is the canonical generator, $\lambda^{2 n}(f)(\xi)$ is Adams e $C^{-i n v a r i a n t .}$

Proof. Adams [2] defines his invariant in this case as follows. Let $u_{q} \in H^{2 q}\left(S^{2 q}\right), u_{n} \in H^{2 n}\left(S^{2 n}\right), \bar{u}_{q} \in H^{2 q}\left(S^{2 n} \cup_{f} e^{2 q}\right)$ and $\bar{u}_{n} \in H^{2 n}\left(S^{2 n} \cup_{f} e^{2 q}\right)$ be generators such that $j^{*}\left(u_{q}\right)=\bar{u}_{q}$ and $i^{*}\left(\bar{u}_{n}\right)=u_{n^{*}}$ Let $\xi_{q} \in \tilde{K}\left(S^{2 q}\right)$ and $\xi_{n} \in \widetilde{K}\left(S^{2 n}\right)$ be the canonical generators and suppose $\operatorname{ch}\left(\xi_{q}\right)=u_{q^{\prime}} \operatorname{ch}\left(\xi_{n}\right)=u_{n}$. If $\xi^{\prime} \in \tilde{K}\left(S^{2 n} \cup e^{2 q}\right)$ is such that $i^{*}\left(\xi^{\prime}\right)=\xi_{n}$, then $\operatorname{ch}\left(\xi^{\prime}\right)^{q}=\bar{u}_{n} \otimes 1+\bar{u}_{q} \otimes \lambda \epsilon$ $\tilde{H}^{*}\left(S^{2 n} \cup_{f} e^{2 q}\right) \otimes Q$. He then defines $\lambda(f)=[\lambda] \in Q / Z$ and shows that $[\lambda]$ is his $e_{C}$-invariant.

From the diagram

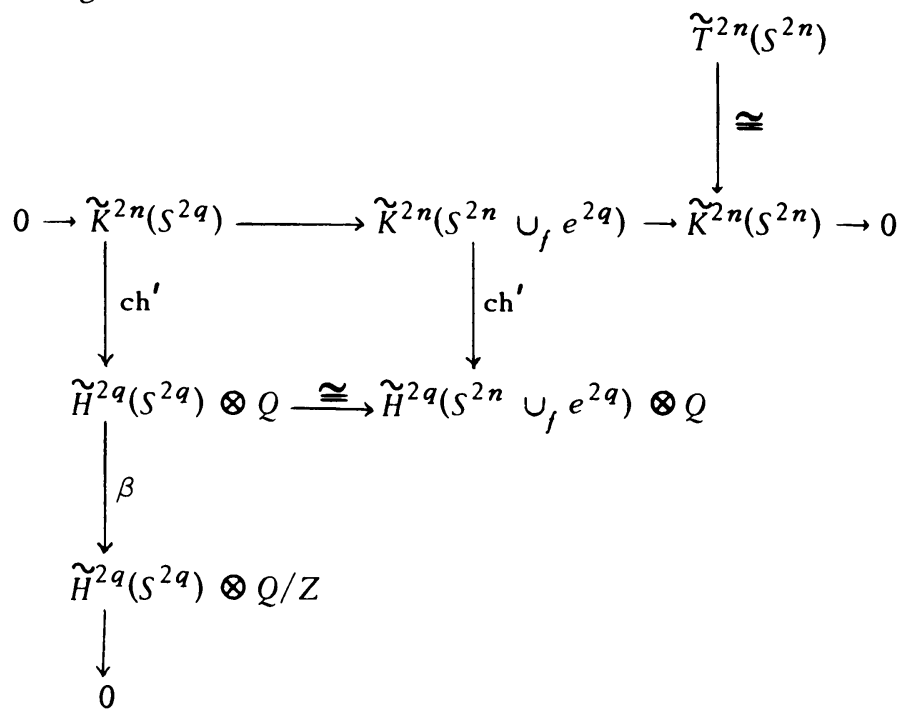

and the fact that $\operatorname{ch}^{\prime}\left(\xi_{q}\right)=u_{q} \otimes 1$ so $\beta=1 \otimes \rho$, we easily see that $\lambda^{2 n}(f)=$ $[\lambda]=\lambda(f)$.

Thus our graded invariant $\left\{e^{n}(f)\right\}$ is essentially Adams' $e_{C}$-invariant.

Adams' $e_{C}$-invariant measures when a split short exact sequence splits with 
respect to the Adams operations $\psi^{k}$. We show that the graded invariant $\left\{e^{n}(f)\right\}$ does the same.

Theorem 7.4. Let $A \stackrel{i}{\rightarrow} X \stackrel{j}{\rightarrow} X / A$ be a cofibration with $\tilde{K}^{*}(A)$ torsion free and $i^{*}: \tilde{K}^{*}(X) \rightarrow \tilde{K}^{*}(A)$ an epimorphism. Then $\tilde{K}^{*}(X) \rightarrow \tilde{K}^{*}(X, A) \rightarrow \tilde{K}^{*}(A)$ splits with respect to the Adams operations if and only if the coboundaries $\delta_{T}^{*}: \tilde{T}^{n}(A)$ $\rightarrow D^{n}(X, A)$ are trivial for all $n$.

Proof. By Proposition 7.1, if $\delta_{T}^{*}: \tilde{T}^{n}(A) \rightarrow D^{n}(X, A)$ is trivial we have a short exact sequence

$$
0 \rightarrow \tilde{T}^{n}(X, A) \stackrel{j^{*}}{\longrightarrow} \tilde{T}^{n}(X) \stackrel{i^{*}}{\longrightarrow} \tilde{T}^{n}(A) \rightarrow 0 .
$$

and, by Theorem 5.7, there is a commutative diagram

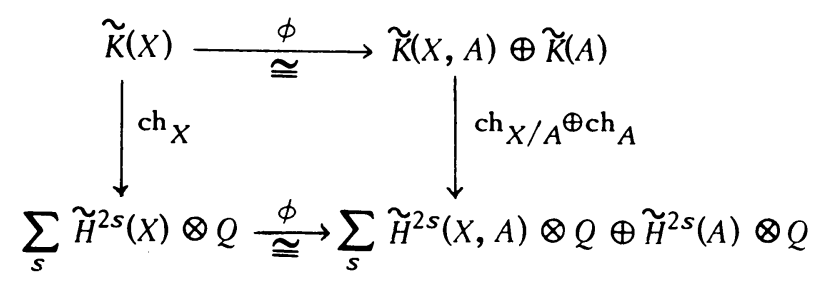

if and only if $i^{*}: \widetilde{T}^{n}(X) \rightarrow \widetilde{T}^{n}(A)$ is an epimorphism for all $n$. Since the Chern character determines the operations $\psi^{k}$ on the torsion free part of $\underset{\sim}{\widetilde{K}}(X), \phi \psi_{X}^{k}=$ $\psi_{X / A}^{k} \oplus \psi_{A}^{k}$ on the torsion free part. Since $j^{*}$ : tors $\underset{\sim}{\widetilde{K}}(X, A) \rightarrow$ tors $\tilde{K}(X)$ is an isomorphism, $\phi\left(\psi_{X}\right)=\psi_{X / A}$ on the torsion part of $\tilde{K}(X)$. Similar remarks are valid for $\tilde{K}^{-1}(X)=\tilde{K}(S X)$.

Corollary 7.5. If $f: X \rightarrow Y$ and $\tilde{K}^{*}(Y)$ is torsion free then $\tilde{K}^{*}(Y \cup, C X) \cong$ $\tilde{K}^{*}(S X) \oplus \tilde{K}^{*}(Y)$ splits with respect to the Adams operations if and only if the graded invariant $\left\{e^{n}(f)\right\}$ is trivial.

In the case $\tilde{K}^{*}(Y)$ has torsion the invariant $\left\{e^{n}(f)\right\}$ fails to give complete information on the splitting of $\tilde{K}^{*}(Y \cup f, C X)$.

We remark that the filtration of $\widetilde{K}(X)$ and $\tilde{K}^{-1}(X)$ by the groups $\tilde{T}^{n}(X)$ enables one to define a graded $d$-invariant as follows. Let $f: X \rightarrow Y$ and set

$$
d^{n}(f)=f^{*}: \widetilde{T}^{n}(Y) \rightarrow \widetilde{T}^{n}(X), \quad \bar{d}^{n}(f)=f^{*}: D^{n}(Y) \rightarrow D^{n}(X) .
$$

Exactly as in Adams [2] one proves elementary propositions relating $\left\{d^{n}\right\}$, $\left\{\bar{d}^{n}\right\}$, and $\left\{e^{n}\right\}$. For example

Proposition 7.6. If $W \stackrel{f}{\rightarrow} X \stackrel{g}{\rightarrow} Y \stackrel{b}{\rightarrow} Z$ and $\left\{d^{n}(g)\right\}=0$ then $e^{n}(g f)$ and $e^{n}(b g)$ are defined and $e^{n}(b g)=e^{n}(g) d^{n}(b)$ and $e^{n}(g f)=\bar{d}^{n}(S f) e^{n}(g)$.

We do not know the interpretation of Toda brackets in the context of these graded $e$-invariants. 


\section{REFERENCES}

1. J. F. Adams, Vector fields on spheres, Ann. of Math. (2) 75 (1962), 603-632. MR $25 \# 2614$.

2. - On the groups $J(X)$. IV, Topology 5 (1966), 21-71. MR $33 \# 6628$.

3. M. Atiyah and F. Hirzebruch, Vector bundles and homogeneous spaces, Proc. Sympos. Pure Math., vol. 3, Amer. Math. Soc., Providence, R. I., 1961, pp. 7-38. MR 25 \#2617.

4. R. Bott, The stable homotopy of the classical groups, Ann. of Math. (2) 70 (1959), 313-337. MR 22 \#987.

5. A. K. Bousfield, A vanishing theorem for the unstable Adams spectral sequence, Topology 9 (1970), 337-344. MR $42 \# 1119$.

6. E. Dyer, Cohomology theories, Math. Lecture Note Series, Benjamin, New York, 1969. MR $42 \# 3780$.

7. B. Eckmann, Systeme von Richtungsfeldern in Sphären und stetige Lösungen komplexer linearer Gleichungen, Comment. Math. Helv. 15 (1943), 1-26. MR 4, 173.

8. I. M. James, $O_{n}$ the homotopy groups of spheres, Sympos. Internacional de Topologia Algebraica, Universidad Nacional Autónoma de México and UNESCO, Mexico City, 1958, PP. 222-224. MR $22 \# 5036$.

9. - Cross-sections of Stiefel manifolds, Proc. London Math. Soc. (3) 8 (1958), 536-547. MR $20 \# 7268$.

10. A. T. Lundell, Homotopy periodicity of the classical Lie groups, Proc. Amer. Math. Soc. 18 (1967), 683-690. MR 37 \#938.

11. — A Bott map for non-stable homotopy of the unitary group, Topology 8 (1969), 209-217. MR 38 \#6595.

12. - An exact sequence involving the Chern character, Bull. Amer. Math. Soc. 77 (1971), 1014-1017. MR $44 \# 3342$.

13. F. Sigrist, Groupes d'homotopy des variétés de Stiefel complexes, Comment Math. Helv. 43 (1968), 121-131. MR 37 \#5876.

14. P. L. Tchebychef, Oeuvres. Tomes I, II, Commissionaires Acad. Impér. Sci., St. Petersburg, 1899-1907; reprint, Chelsea, New York, 1962. MR 26 \#4870.

DEPARTMENT OF MATHEMATICS, UNIVERSITY OF COLORADO, BOULDER, COLORADO 80302 